\title{
Extraction and Visual Analysis of Potential Vorticity Banners around the Alps
}

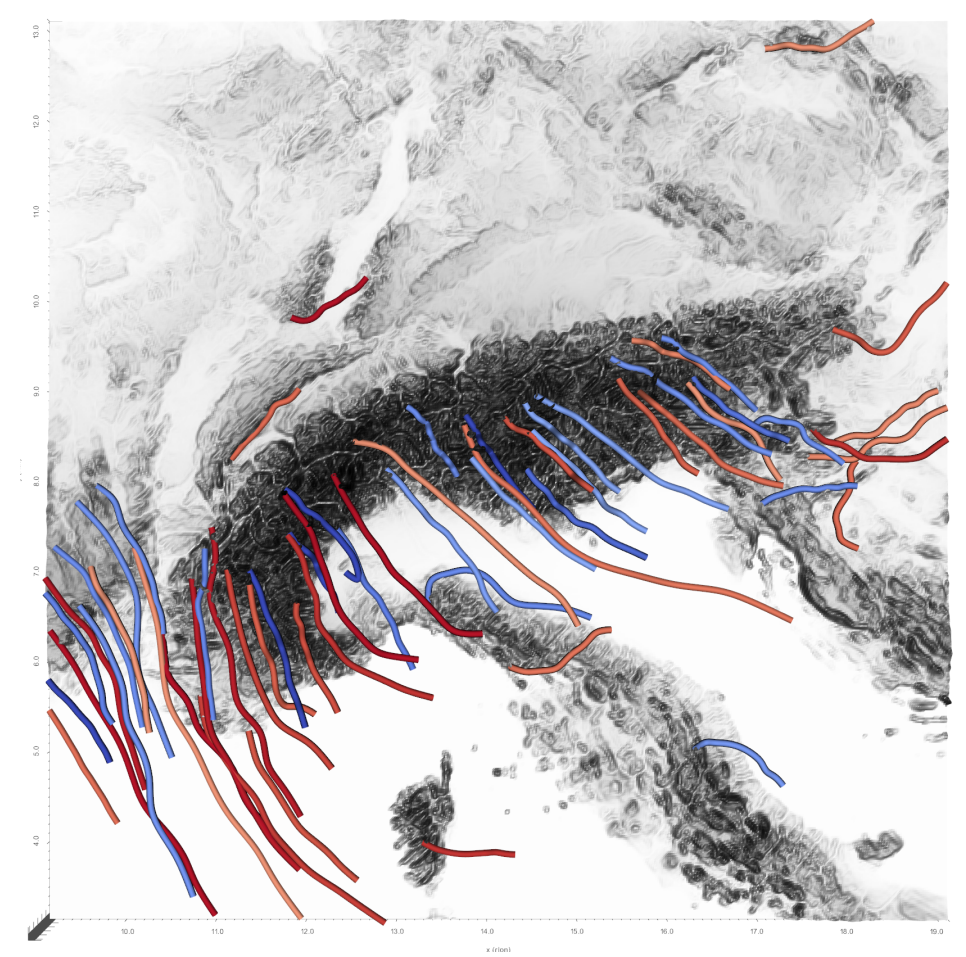

Robin Bader

Master Thesis

April 2019

Thesis Project Mentor

Prof. Dr. Markus Gross

Supervised by

Dr. Tobias Günther

\section{EHH}





\section{Abstract}

Potential vorticity (PV) is a quantity that can be computed by combining the rotation and stratification in the atmosphere. Due to the combination of these physical processes potential vorticity is considered as one of the most important scalar quantities in atmospheric dynamics. It is able to explain the occurrence of frontal rain bands and plays a key role in particularly strong wind peaks in extratropical cyclones. When wind flows around mountain peaks, rather than over it, a potential vorticity accumulation shaped as elongated banners forms in the mountain's lee. The role of these so called potential vorticity banners has recently raised considerable interest in the meteorological community for instance due to their influence in aviation wind hazards and maritime transport.

In order to support meteorologists and climatologists in the analysis of these structures, we developed an extraction algorithm and a visual exploration framework consisting of multiple linked views. For the extraction of the PV banners we apply a predictor-corrector algorithm that follows streamlines and realigns them with extremal lines of potential vorticity. Using the agglomerative hierarchical clustering algorithm, we group banners from different sources based on their proximity. To visually analyze the time-dependent banner geometry, we provide interactive overviews and enable the query for detail on demand, including the analysis of different time steps, potentially correlated scalar quantities, and the wind vector field.

In particular, we study the relationship between relative humidity and the banners for their potential in indicating the development of precipitation. Working with our method, the collaborating meteorologists gained a deeper understanding of the three-dimensional processes, which may spur follow-up research in the future. 



\title{
EIH
}

Eidgenössische Technische Hochschule Zürich

Swiss Federal Institute of Technology Zurich

\author{
Master Thesis
}

\section{Visualization and Extraction of Potential Vorticity Banners Around the Alps}

\section{Project Description}

The Institute of Atmosphere and Climate Research (IAC) at ETH performs high-resolution simulations of atmospheric air flows in and around the Alps. At present, the meteorological attributes are studied mainly with straightforward 2D plots at certain height levels. Many atmospheric processes, however, are inherently three-dimensional such as the creation of potential vorticity (PV) banners, which are tightly coupled to wind, temperature and pressure. In order to identify the meteorological conditions that lead to the creation and temporal evolution of PV banners we investigate their structure in 3D and correlate them with meteorological attributes such as temperature, relative humidity and others. We investigate the air flow around PV banners using trajectory information which leads us to a combination of Eulerian properties (observations at grid points) and Lagrangian properties (computed from or integrated along particle trajectories). The work is done in close collaboration with domain scientists from the Institute of Atmosphere and Climate Research.

\section{Tasks}

To enable the analysis of the 3D structures in numerical weather data a prototype is being developed using the Visualization Toolkit (VTK) that allows the integration of the data provided by the IAC. This includes interactive rendering, correct handling of height information and the advection of particles. The meteorologists and climatologists at the IAC provide continuous support and feedback in the development process of the prototype. Through a continuous adaption of the requirements of the prototype an application is developed which meets their demands to facilitate the analysis of meteorological data.

This led to a number of possible avenues that this thesis can take, including the robust extraction of PV banners, their visualization in 3D and the correlation with other meteorological attributes. To extract PV banners different methods are evaluated, which includes a region-based extraction and a feature line extraction to determine their corelines. We further consider the investigation of trajectory data along corelines which requires a visual abstraction, hierarchical clustering, multi-field encoding of scalar values, and interactive linking and brushing. The investigation of PV corelines over time creates further challenges regarding temporal coherence, which should be considered. The feature extraction and visualization algorithms are tested on a numerical weather prediction time series and are evaluated in coordination with the domain scientists to assess the usability and the possible insights that might be gained by the interactive visualization in 3D.

\section{Remarks}

A written report and an oral presentation conclude the thesis. The thesis will be overseen by Prof. Markus Gross and is supervised by Dr. Tobias Günther.

\section{Project}

Start: 31. October 2018

End: 30 . April 2019 



\section{Acknowledgment}

I thankfully look back at a great experience while working on this Thesis.

I particularly want to thank my supervisor Dr. Tobias Günther who supported me while I was working on this Master Thesis. I am very grateful to Tobias's challenging questions and valuable advise which played a central role to complete this work. I learned a lot from our respectful, open and friendly interactions we had.

Further I want to thank Dr. Michael Sprenger, Dr. Nikolina Ban, Dr. Stefan Rüdisühli and Prof. Dr. Christoph Schär from the Institute for Atmospheric and Climate Sciences at ETH Zurich who supported me in every aspect they could. Through regular meetings they enabled me an insight into their meteorological work and supported me in every question I had.

Finally, I express my gratitude to my parents and friends who have supported me during all these years. 



\section{Contents}

List of Figures $\quad$ ix

List of Tables $\quad$ xiii

$\begin{array}{ll}\text { 1. Introduction } & 1\end{array}$

2. Related Work 5

2.1. Definition of Potential Vorticity . . . . . . . . . . . . . . 5

2.2. Potential Vorticity Banners . . . . . . . . . . . . . . . 6

2.3. Feature Line Extraction . . . . . . . . . . . . . . . . . . . . 7

2.4. Meteorological Visualization . . . . . . . . . . . . . . 8

3. Method 11

3.1. Requirements . . . . . . . . . . . . . . . . . . 11

3.2. Method Overview . . . . . . . . . . . . . . . . . . . . 12

3.3. Extraction of Potential Vorticity Banners . . . . . . . . . . . . . . . . . 12

3.3.1. Predictor-Corrector Algorithm . . . . . . . . . . . . . . . 13

3.3.2. Seed Points . . . . . . . . . . . . . . . . . . . . 15

3.3.3. Banner Termination \& Filtering . . . . . . . . . . . . . . 15

3.4. Clustering of Potential Vorticity Banners . . . . . . . . . . . . . . . 15

3.4.1. Distance Metric . . . . . . . . . . . . . . . . . . . . . . 15

3.4.2. Spatial Clustering . . . . . . . . . . . . . . . . . . . . . . . . . . . . 16

3.4.3. Temporal Clustering . . . . . . . . . . . . . . . . . . . . . . . . . . . . . . . . . 17

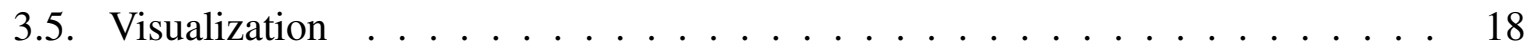

3.5.1. Geospatial View . . . . . . . . . . . . . . . . 18

3.5.2. Multi-Variate View . . . . . . . . . . . . . . . . . . . . . . . 19

3.5.3. Time-Series View . . . . . . . . . . . . . . . . . . . . . . 20

3.5.4. Node-based Editor . . . . . . . . . . . . . . . . . 21 
4. Implementation 23

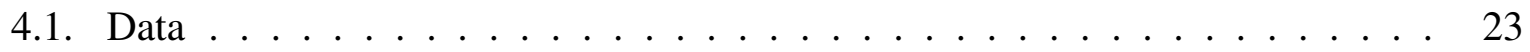

4.2. Details to numerical data . . . . . . . . . . . . . . . . 24

4.2.1. Cell Sampling . . . . . . . . . . . . . . . . . 25

4.2.2. Cell Indexing in Surface Aligned Grid . . . . . . . . . . . . . . . . . . . . . 26

4.2.3. Rotated Longitude/Latitude Grid . . . . . . . . . . . . . . . . . . . 27

4.2.4. Calculation of distances in Spherical Earth . . . . . . . . . . . . . . . 28

4.2.5. Finite Differences in Surface Aligned Grid . . . . . . . . . . . . . . . 29

4.2.6. Converting from geographical altitude to Index . . . . . . . . . . . . . 30

4.2.7. Interpolation . . . . . . . . . . . . . . . . 30

4.3. Software Architecture . . . . . . . . . . . . . . . . . 31

4.3.1. Presentation-Layer . . . . . . . . . . . . . . . . . 32

4.3.2. Processing-Layer . . . . . . . . . . . . . . 33

4.3.3. Data-Layer . . . . . . . . . . . . . . . . 34

$\begin{array}{ll}\text { 5. Results } & 37\end{array}$

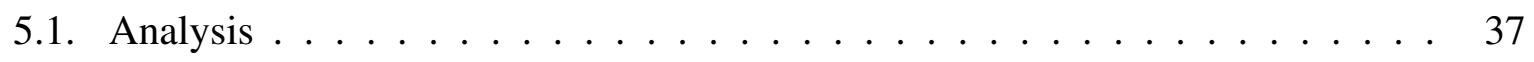

5.2. Development Process \& Design Lessons . . . . . . . . . . . . . . . . . . . . . 40

5.3. Performance ......................... 40

6. Conclusion and Future Work 45

$\begin{array}{ll}\text { A. Appendix } & 47\end{array}$

A.1. Filter, algorithms and node types of application $\ldots \ldots \ldots$. . . . . . . 47

$\begin{array}{ll}\text { Bibliography } & 50\end{array}$ 


\section{List of Figures}

1.1. Traditional visualization of potential vorticity as $2 \mathrm{D}$ slice, computed on a fixed altitude level. Potential vorticity (PV) banners are created during the detachment of flow from the mountain topography and can extend several hundred $\mathrm{km}$ downstream. Here, the banners appear in the lee of the Alps as line-shaped regions with significantly negative (blue) or positive (red) PV values. . . . . . .

1.2. Our line extraction allows us to depict banners over different altitude levels and extracts only PV banners that originate from flow detachment at the topography. (a) Visualization of extracted PV banners clustered into groups depending on their spatial proximity. (b) Visualization of our PV banner lines in 3D before clustering into banner representatives. Our method allows for the interpretation of the vertical extent of the banners, reduces visual clutter and shows PV banners across all altitude levels. . . . . . . . . . . . . . . . . .

2.1. (a) PV banners form in the lee of a mountain when air flows around rather than over it. Primary banners, colored in red (positive PV) and blue (negative $\mathrm{PV}$ ), are on the scale of the whole mountain. Their width is determined by boundary layer processes and turbulence. The width of secondary banners, which are created in the lee of individual peaks and massifs, is determined by the upstream topography. (b) The rotation direction of a potential vorticity banner depends on which side the air flows around the mountain.

2.2. Vertical cross section of secondary banners in a numerically-simulated flow, showing the velocity using an arrow plot projected onto a plane. The color denotes the PV value at each point and the arrows highlight the swirling motion. 
3.1. Method overview. (a) The wind velocity and potential vorticity scalar field serve as inputs to our algorithm. (b) The PV banner extraction algorithm distributes seed points along the mountain surface and integrates the banners using a predictor-corrector algorithm. (c) Using a similarity matrix, we cluster similar banners into groups and determine the representatives. (d) Using multiple linked views, a flexible node-based editor and interactive filtering, we can highlight the desired properties of the banners. (e) Users interact with all views by picking as well as linking and brushing. . . . . . . . . . . . . . .

3.2. Illustration of the correction step of the predictor-corrector algorithm, which corrects a predicted point by setting it to the potential vorticity minimum (maximum) using the method of steepest descent (ascent). Projecting the gradient on a plane approximately parallel to the velocity allows us to find the extremum at the intersection of the implicit lines where the gradient of each component is

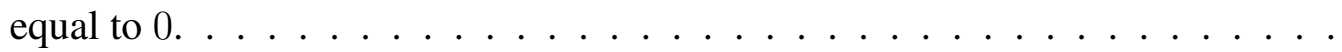

3.3. Cluster algorithm using AHC. The grid represents a spatial data structure. Lines that share a grid cell are compared with each other, e.g., the blue or green shaded cells highlight overlapping line elements in the spatial grid. $s_{1}$ and $s_{2}$ have a high similarity measure and are clustered together. None of the lines have a high similarity with $s_{3}$ and are therefore clustered independently. . . . . . .

3.4. The PV banners are colored based on their cluster membership. This enables lines to be categorized together that are integrated from different seed points but converged into the same banner. We apply the AHC to group banners and select representative lines. . . . . . . . . . . . . . . .

3.5. (a) In order to observe the temporal evolution of PV banners, we combine the closest lines between time step $t_{i}$ and $t_{i+1}$ in a temporal structure. (b) Example of the temporal banner tracking, when following a selection of PV banners 4 hours in time (24 time steps). The selection stays stable for the banners. The green banner dissolved over time. . . . . . . . . . . . . . .

3.6. Time-dependent projection of the PV banners length onto their seed points. Enables perception of geographical important banner seed points. The long banners in the east are part of a primary PV banner in the scale of the whole Alps. Several high peaks (magnified) show an increased banner length compared to their surroundings. Time period: 29 January 2010 00:00 to 08:00 UTC. . . . . . . .

3.7. Example of linked views. (a) Parallel Coordinates Diagram. Selection of positive PV banners with low updraft. Selection automatically updates all other views. (b) Updated view according to selection in (a). (c) Limit selection by placing shapes within the scene. E.g., box that filters banners outside of it. . . . . . . .

3.8. Data flow programming implementation. Enables a high degree of flexibility by defining combining operators through connections while hiding the underlying algorithmic complexity. This specific example samples seed points along the surface, integrates the banners using the predictor-corrector algorithm, filters the banners that are within a user defined box and shows the temporal accumulation of them in the view using the depicted color maps. . . . . . . . . . . . . 
4.1. Schematic overview of subset extraction. In the three dimensional grid a horizontal subset can optionally be extracted to increase loading times and optimize memory footprint. . . . . . . . . . . . . . . . . . .

4.2. Schematic overview of cell sampling onto half levels or the cell's center. Depending on the resolution in the vertical the data is sampled in different locations. 27

4.3. Surface aligned grid where the data is sampled in higher resolutions along the surface which. This data structure optimizes the storage requirements to persist the data. The visualization emphasizes the irregularity of the levels altitudes shown as lines

4.4. Indexing in surface aligned grid. The vertical index $z=0$ refers to the highest level in the altitude.

4.5. Comparison of cropped subregion used to present the results of this work with the original datasets extent. (a) Full extent of provided data in rotated longitude and latitude coordinate system. (b) Cropped subregion of simulation data over the Swiss Alps used in project. . . . . . . . . . . . . . .

4.6. Visualization of the altitude conversion algorithm. Given the half level sampled altitudes we first derive the cell center's altitudes. We want to find the index that maps the data to a point $(x, y, z)$ in the model's atmosphere. The algorithm searches for this point in the ordered altitude set by finding the cell center whose altitude is above the $z$. This enables us to interpolate the index. . . . . . . . . .

4.7. To interpolate the values in the irregular grid we need to first interpolate the vertical components on a horizontal plane and then use bilinear interpolation.

4.8. The presentation-, processing- and data- layers build the foundation of the software architecture. . . . . . . . . . . . . . . . . .

4.9. The data flow in the directed graph of the node based editor is processed from input- to output-nodes.

4.10. (a) Using a scatter plot matrix we can select the PV values in cells that also have rain. (b) The selected PV values are visualized using a volume rendering. . . . .

5.1. PV value along the extracted core lines. The absolute PV value declines with the length of the banner. Typically the neighboring PV banners with anomalously positive (red) and negative (blue) signs show similar trends along their length. The mean is shown as a black line. . . . . . . . . . . . . .

5.2. Distribution of PV in the model's atmosphere sampled in cells of constant volume in the altitude range of $0 \mathrm{~km}$ to $6 \mathrm{~km}$ above sea level. The mean of the normally distributed PV values is slightly shifted to the positive side, which was expected by our domain scientists. . . . . . . . . . . . . .

5.3. (a) Selected PV banners extracted to analyze their spatial extent. (b) Horizontal cross section of the vertical wind component at fixed altitude along the PV banner. The wave-like patterns in the flow direction (from left to right) is associated with atmospheric gravity (or buoyancy) waves and is responsible for the altitude changes of PV banners. (c) Altitude of the PV banners along their full extent. The banners show an undulating pattern in the altitude change close to their source. Color-coded positive (red) and negative (blue) banners. . . . . . . . . . 
5.4. Relative humidity extracted as a horizontal cross section around the PV banners. A correlation between the PV banners and relative humidity values is visible. This highlights that PV banners separate air masses of alternating high and low humidity.

5.5. PV banners color-mapped by altitude. Banners at different altitudes exhibit different directions and may cross each other. Comprehending these crossings is straightforward in our 3D visualization, but difficult to detect in standard horizontal displays. 27 January $201020: 10$ UTC. . . . . . . . . . . . . . . . . . .

5.6. 29 January 2010 from 00:00 to 05:00 UTC. End of lifetime sequence of PV banners which are rendered as volumes with the corresponding core lines as geometrical lines. (a) The wind flows from north (left) to south (right). The wind direction in the Po valley changes eastwards which is responsible for the eastward bend in their end. (b) The PV banner start to decouple from the mountains source. (c) The decoupled PV banners are advected with the wind flowing over the Alps. (d) Horizontal cross section of the PV banners on $3000 \mathrm{~m}$ above sea level which emphasizes the decoupling of the PV banners. . . . . . 


\section{List of Tables}

4.1. Listing of scalar values required to extract PV banners and generate the presented results. . . . . . . . . . . . . . . . . . . . 26

5.1. The timings were measured on a grid with $500 \times 500 \times 60$ resolution, based on the node graph earlier shown in Figure 3.8. . . . . . . . . . . . . . . . 41

A.1. List of the implemented node types in application. Input \& output describe the datatype of the nodes. . . . . . . . . . . . . . . . . . . . 



\section{1}

\section{Introduction}

Meteorologists face the challenge to process and interpret data with high temporal and spatial variability. Understanding this data enables the research and development of forecasting techniques which finally improves the quality of weather forecasts. Traditional analyses of numerical weather simulation data and their features focus on the two-dimensional extraction of slices along with certain altitude levels. However, many meteorological processes, such as the formation of clouds and precipitation, are inherently three-dimensional and exhibit a high temporal and spatial variability. Meteorologists are required to understand these processes since they are not only crucial for short-term numerical weather prediction, but also for long-term climate change simulations. In the challenging environment of ever-increasing data accuracy, spatial and temporal resolution $\left[\mathrm{SKV}^{+} 17, \mathrm{LLB}^{+} 17, \mathrm{SANB}^{+} 19\right]$ the meteorologists require a tool that facilitates their research by enabling them deeper insight into their data.

One of the main goals of synoptic meteorology is to identify the key drivers of weather evolution and their potential interaction. Within the continuous basic meteorological fields (e.g., wind speed or temperature) distinct meteorological features are embedded and can be identified. For instance, well-known embedded Eulerian features are extratropical cyclones and jet streams, and an example for a Lagrangian feature is the warm-conveyor belt [SFB $\left.{ }^{+} 17\right]$. In this work, we focus on a less familiar mesoscale feature, namely on so-called potential vorticity (PV) banners in the lee of the European Alps.

Potential vorticity is an embedded feature that combines the rotation and stratification in the atmosphere. When the synoptic-scale wind turns into a direction crossing the Alpine ridge, low-level elongated accumulations of anomalously positive and negative PV values form [AS98, $\left.\mathrm{BSB}^{+} 02\right]$. These so-called PV banners can be attributed to flow splitting, either on the scale of the whole Alps (primary banners) or on that of individual massifs and mountain peaks (secondary banners). The existence of PV banners has initially been proposed based on numerical experiments [AS98] and later been confirmed in field experiments using multiple research aircrafts $\left[\mathrm{BBB}^{+} 01, \mathrm{SSL}^{+} 03\right]$. Figure 1.1 displays a conventional 2D slice of potential vorticity in 


\section{Introduction}

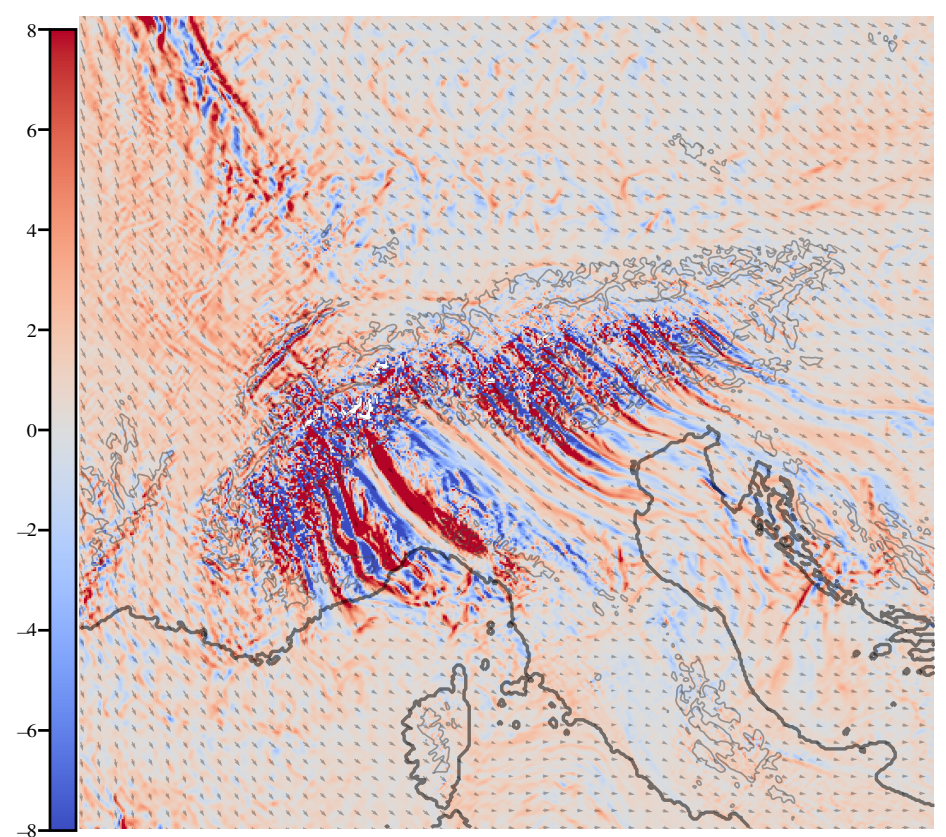

Figure 1.1.: Traditional visualization of potential vorticity as $2 D$ slice, computed on a fixed altitude level. Potential vorticity $(P V)$ banners are created during the detachment of flow from the mountain topography and can extend several hundred $\mathrm{km}$ downstream. Here, the banners appear in the lee of the Alps as line-shaped regions with significantly negative (blue) or positive (red) PV values.

the atmosphere above the Alps and Figure 1.2 shows our interactive visualization of the extracted 3D PV banners.

These banners interactions are key drivers in various meteorological processes. The primary PV banners are jointly responsible for the development of cyclones in the lee of the Alps, see e.g., Kljun et al. [KSS01] and references therein. In secondary PV banners, an inertial (or symmetric) instability is released in the negative PV banner [Hos74]. Negative PV (and symmetric instability) is assumed to play a key role in particularly strong wind peaks embedded in extratropical cyclones [VCG18]. It was also hypothesized that the symmetric instability is able to explain the occurrence of elongated rain bands [BH79] - an idea that was recently further discussed by Schumacher et al. [SSK10] for convective snow bands downstream of the Rocky Mountains and by Siedersleben and Gohm [SG16] for Alpine flows.

In order to follow up on the research of PV banners, we use our visualizations and extraction to investigate in two cases the role of PV banners with their surrounding.

This work has been motivated by the requirement of meteorologists to gain a deeper understanding of these three dimensional structures and their sometimes short-lived presence. Due to the high resolution of the numerical weather prediction (NWP) data and the high dimensionality of its space-time domain [RBS $\left.{ }^{+} 18\right]$ an effective visual analysis of the dynamic behavior of PV banners is a challenging problem. Understanding their creation and evolution is a multi-field visualization problem since the presence of banners is linked to other meteorological attributes. The cores of PV banners are temporally moving and deforming, multiple PV banners may be in very close proximity, and the numerical data is highly anisotropically sampled. Through close 


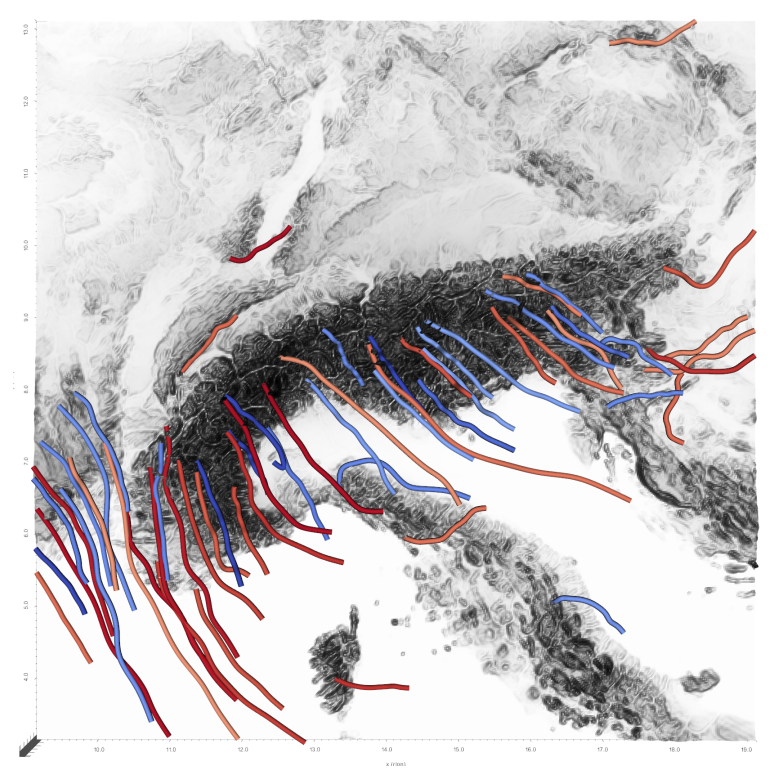

(a)

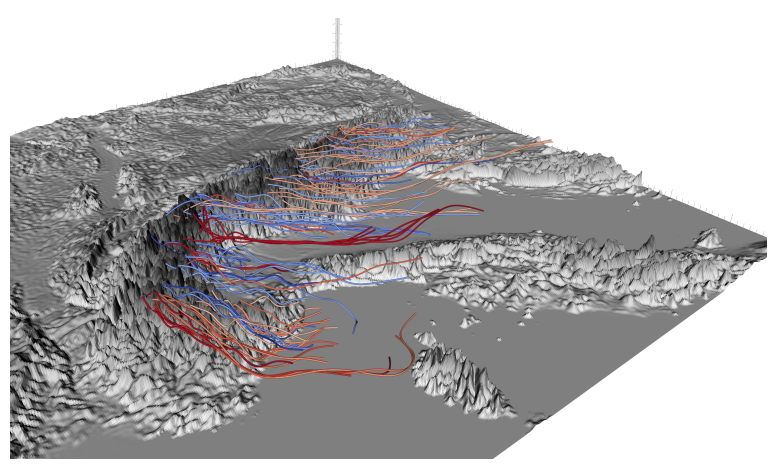

(b)

Figure 1.2.: Our line extraction allows us to depict banners over different altitude levels and extracts only PV banners that originate from flow detachment at the topography. (a) Visualization of extracted $P V$ banners clustered into groups depending on their spatial proximity. (b) Visualization of our $P V$ banner lines in $3 D$ before clustering into banner representatives. Our method allows for the interpretation of the vertical extent of the banners, reduces visual clutter and shows $P V$ banners across all altitude levels.

collaboration with four domain scientists we derived an automatic feature extraction approach that computes a geometric line representing each banner (See Figure1.2(a) and (b)). From the geometric representation, we extract curve characteristics (length, curvature, lifetime, etc.) which can be interactively explored alongside given meteorological attributes (i.e., relative humidity) in order to study correlations. Our system consists of three components:

- Feature Extraction. We develop an automatic predictor-corrector algorithm that is initialized on the mountains and uses the wind vector field and the potential vorticity's gradient to extract the core lines of minimal and maximal potential vorticity.

- Clustering. We use agglomerative hierarchical clustering to remove duplicate PV core lines. This enables the observation of their temporal life cycle and to distinguish their origins.

- Visualization. We provide multiple linked views that display the spatial embedding in 3D and the time-dependent evolution of attributes and properties along the curves. Our user interface provides a flexible node-based editor that allows for interactive filtering, linking and brushing.

We extract and visualize the PV banners in two high-resolution numerical simulations, demonstrating the effectiveness of the visualization system in explaining the inherently threedimensional processes of PV creation and evolution, and their relationship with meteorological attributes. In particular, we identify two phenomena that our domain scientists were interested in and are intractable to comprehend from conventional 2D slices: cross flows of PV banners in 


\section{Introduction}

different altitudes and the vertical waviness of PV banners.

In Chapter 2 of this document, we introduce the definition of potential vorticity and review previous work on the analysis of PV banners. We provide an overview of different line extraction methods and introduce their advantages and disadvantages. Finally, we review recent work in meteorological visualization in three dimensions.

Chapter 3 introduces the requirements that the developed application needs to comply in order to facilitate the research on PV banners. Based on these requirements the extraction method was developed which includes the predictor-corrector algorithm, the placement of seed points and the clustering of PV banners. Finally, the visualization framework is presented which introduces the different visualization types and their applications within the developed framework.

Details to the implementation of the method and the visualizations are given in Chapter 4 . The mathematical and algorithmic foundations to work with the challenging geographical dataset are introduced. This is followed by detailing the software architecture that enables flexible integration of the method by abstracting the problem into sub-layers.

Chapter 5 gives insight into the results gained throughout this work including an analysis of the datasets, development lessons and performance evaluation.

Finally, Chapter 6 concludes the current work with a summary of the work and possible future extensions and improvements. 


\section{2}

\section{Related Work}

This chapter introduces the definition of potential vorticity and explores related work on PV banners in the meteorological community. By introducing different feature extraction methods we give an overview of previously done extractions with a focus on vorticity coreline extraction. Finally, an overview of the current research done in meteorological visualization is given.

\subsection{Definition of Potential Vorticity}

Potential vorticity is a simplified approach to understand the fluid motions in a rotating system such as the Earth's atmosphere and ocean. It describes the rotation of an air parcel between levels of constant potential temperature (isentropic levels) by embedding a signed scalar value that is derived from vorticity. The resulting quantity can only be changed by diabatic or frictional processes which conserve an air parcel's PV during advection [Ert42, HMR85]. Since PV is invariant to an air parcel's change in position and altitude, it, therefore, behaves under adiabatic and frictionless conditions as a tracer, i.e., it is advected along by the ambient flow field.

Note, however, that PV is not passively advected by the wind field, as is the case, for instance, for a dye introduced in water. Rather, by means of the invertibility principle, $\mathrm{PV}$ is able to influence the ambient flow field itself [HMR85]. This behavior as an active, in contrast to a passive tracer, leads to fascinating meteorological features, of which Diabatic Rossby Waves are one specific example [BW11].

Potential vorticity is proportional to the dot product of the absolute vorticity vector (Equation 2.1) and the three-dimensional gradient of potential temperature (Equation 2.2). In the following, we introduce these two quantities.

The absolute vorticity $\zeta_{a}$ is computed from the air velocity $\mathbf{v}$ defined relative to a reference frame rotating with the Earth. Therefore, it includes the Coriolis parameter $f=2 \Omega \sin (\varphi)$ applied to 


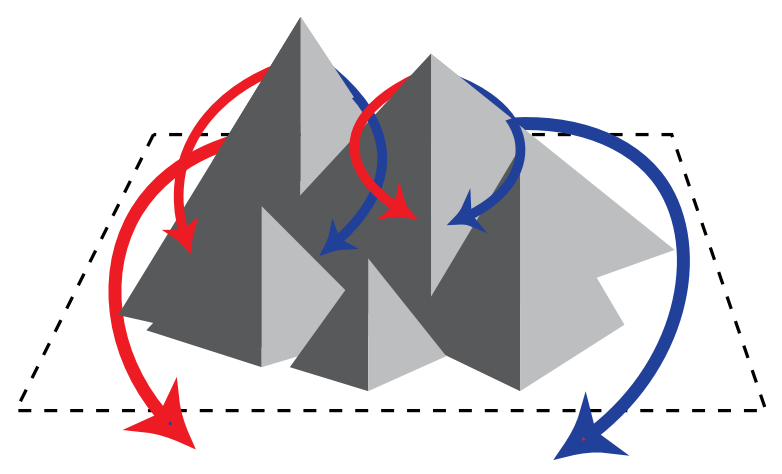

(a)

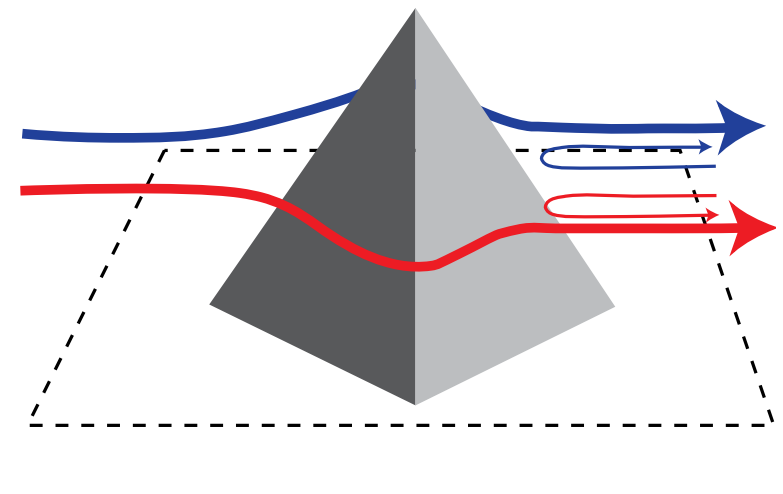

(b)

Figure 2.1.: (a) $P V$ banners form in the lee of a mountain when air flows around rather than over it. Primary banners, colored in red (positive $P V$ ) and blue (negative $P V$ ), are on the scale of the whole mountain. Their width is determined by boundary layer processes and turbulence. The width of secondary banners, which are created in the lee of individual peaks and massifs, is determined by the upstream topography. (b) The rotation direction of a potential vorticity banner depends on which side the air flows around the mountain.

the unit vector $\mathrm{k}$ in vertical direction, where $\Omega=7.29 \cdot 10^{-5} \mathrm{rad} / \mathrm{s}$ is the angular velocity of the Earth [Wik19], and $\varphi$ the latitude:

$$
\zeta_{a}=\nabla \times \mathbf{v}+f \times \mathbf{k}
$$

where $\mathbf{k}$ denotes the vertical unit vector. The absolute vorticity of an air parcel will change if it is stretched (or compressed) in the vertical direction, but the potential vorticity is conserved in adiabatic and inviscid flows.

The potential temperature $\theta$ of an air parcel at pressure $p$ is the temperature that the parcel would attain if adiabatically brought to a standard reference pressure $p_{0}$. It is given by

$$
\theta=T\left(\frac{p_{0}}{p}\right)^{R / c_{p}}
$$

where $T, R, c_{p}$ are the temperature, gas constants, and specific heat capacity at constant pressure, respectively. The ratio $R / c_{p}=0.286$ is a constant for dry air.

Finally, the potential vorticity $Q$ is defined following Ertel [Ert42] as:

$$
Q=\frac{\zeta_{a} \cdot \nabla \theta}{\rho}
$$

where $\rho$ is the density of the air parcel and $Q$ is denoted by potential vorticity units $(P V U)$ which are defined as $1 \mathrm{PVU}=\frac{10^{-6} \cdot \mathrm{K} \cdot \mathrm{m}^{2}}{\mathrm{~kg} \cdot \mathrm{s}}$.

\subsection{Potential Vorticity Banners}

When synoptic-scale wind turns into a direction crossing the Alpine ridge, low-level elongated bands of potential vorticity form [AS98, $\left.\mathrm{BSB}^{+} 02\right]$ as visible in Figure 1.1 and Figure 1.2. These, 


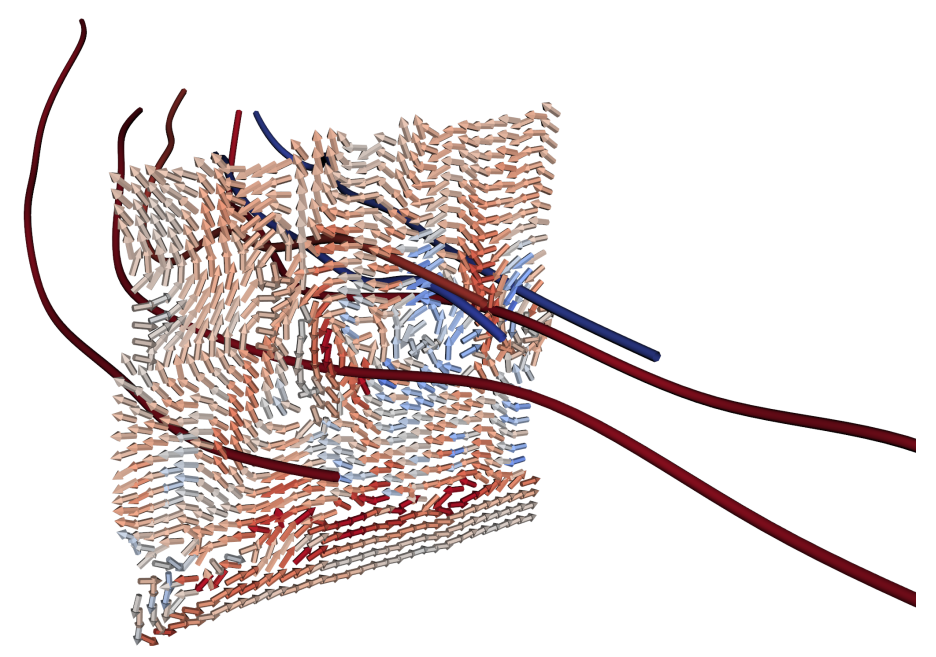

Figure 2.2.: Vertical cross section of secondary banners in a numerically-simulated flow, showing the velocity using an arrow plot projected onto a plane. The color denotes the $P V$ value at each point and the arrows highlight the swirling motion.

so-called PV banners, are represented as structures with low or high PV value and they can be attributed to flow splitting, either on the scale of the whole of the Alps (primary banners), or on that of individual massifs and peaks of the model topography (secondary banners) as schematically visualized in Figure 2.1 (a).

These PV banners are created by air parcels rotating around a vertical common axis along the banner's core line, which may be straight or curved. Figure 2.2 shows the vertical velocity profile which highlights the swirling motion around the core line.

The rotation direction depends on which side the air flows around the peak, as illustrated in Figure 2.1 (b). The banners encompass the wake of the topographic obstacle (i.e., a region of decelerated flow), either on the scale of the whole obstacle (primary banners), or on the scale of individual massifs and peaks (secondary banners).

\subsection{Feature Line Extraction}

Extracting the extremal lines of potential vorticity as a geometric representation of the banner center is particularly useful because it removes visual occlusion and clutter, provides a concise picture of the structure of PV banners, and it enables the observation of the dynamics of meteorological processes along the lines.

Extremal lines are features of a 3D scalar field along which the scalar function becomes minimal or maximal with respect to a local neighborhood [Ebe12]. The extraction of extremal lines has been subject to much research (see Kindlmann et al. $\left[\mathrm{KCH}^{+} 18\right]$ ), and found numerous applications in the extraction of jet-stream core lines [KHS $\left.{ }^{+} 18\right]$, in the extraction of vortex core lines using maximum lines of Q-criterion [SWTH07], minimum lines of pressure [MK97] or 
maximum lines of vorticity magnitude [SKA99]. Peikert and Roth [PR99] introduced the parallel vectors operator and showed that many of the above feature line definitions can be expressed as the union of points at which two vector fields are parallel.

Extremal line extraction algorithms can be categorized into local and integration-based methods. Local methods subdivide the domain into cells, solve for parallel vector points on each cell boundary independently and finally connect the results [HTWS07, GST16].

Banks and Singer [BS94] presented a predictor-corrector method to find vortex core lines, which forms the basis of our extraction algorithm. This integration-based method is more robust in avoiding typically spurious results induced by local methods.

The predictor-corrector algorithm repeats the following three steps: First, take a step along the vorticity vector to move forward. Second, evaluate the vorticity vector at the new position and construct the normal plane. Third, in this plane, correct the predicted point by setting it to the nearest local pressure minimum. A seed point is found by performing a number of initial steps to establish convergence. This procedure is based on the assumption that the direction of the vorticity vector has little variation compared to the variation of pressure. Under this assumption and for small step sizes, the algorithm yields points where vorticity is approximately parallel to the pressure gradient.

Ann integration-based method such as the predictor-corrector method or feature flow fields [TWHS03] requires careful placement of seed points and subsequent removal of duplicates, which we discuss later.

\subsection{Meteorological Visualization}

Meteorological Visualization has a long history and typical tasks include the analysis of data to understand the weather situation or a specific atmospheric process, decision making, and the communication of forecasts and research results [RBS $\left.{ }^{+} 18\right]$. Nocke et al. [NSW99] provide a situation analysis of visualization techniques for climate data by presenting the results of a questionnaire by climate impact researchers using visualization. The biggest identified challenges in meteorological visualization are the heterogeneity of climate data (e.g., the spatial temporal, multi-variate, gridded data) and time-series which require alternative yet intuitive visualization techniques. The questionnaire shows that standard 2D presentation techniques are most frequently used while 3D visualization techniques are of minor importance only. The most typical 3D visualization techniques used for scalar data are isosurfaces, direct volume rendering, realistic rendering and decomposition methods. For vector data, 3D icons and field lines are the preferred techniques.

Trafton and Hoffman [TH07] discussed activities in cognitive engineering to improve meteorological display technology. They list ingrained traditions in meteorological symbology such as wind barbs or isopressure lines which were standardized long ago and identify changing these long-standing traditions as a challenge. One of the requirements for new tools that support mental-model formation is the provision of better spatiotemporal understanding.

Szoke et al. [SGMM03] analyzed the use of a 3D visualization framework in the meteorological 
community and identified a reluctance between forecasters to shift to $3 \mathrm{D}$ visualizations. They identified as the root of this reluctance the enormous shift in how forecasters prepare their forecasts and that meteorologists are trained using traditional 2D approaches. It would require more training to understand and use 3D visualizations. However, they identified several chances that the $3 \mathrm{D}$ visualization has over traditional methods including that meteorologists have less chance to miss some critical features due to missing the examination of the "correct" 2D level or the most appropriate cross section. Furthermore, the interactivity provided in 3D applications was well received and tools such as interactively movable vertical cross-sections, for example, were very well perceived.

A thorough overview of visualization techniques in meteorology can be found in the survey article by Rautenhaus et al. [RBS $\left.{ }^{+} 18\right]$.

With respect to meteorological visualization, our approach is related to the jet-stream core line extraction approach by Kern et al. [KHS $\left.{ }^{+} 18\right]$. They used a local method to extract jet-streams as maximal velocity extremal lines and made the observation that classical local methods commonly produce disjoint and cluttered results. To be more robust they exploit the velocity's directional information by minimizing its magnitude's gradient on an orthogonal plane.

Our extraction method inherits this concept of exploiting the directional information. As done by Kern et al. $\left[\mathrm{KHS}^{+} 18\right]$ we use a, to the velocity, approximately perpendicular plane to find the local extrema. Kern et al. detected the PV points locally and connected them in a postprocess. However, PV banners are extremely thin structures and the numerical data contains small variations, due to the sampling density or noise, which makes it difficult for local methods to produce continuous lines in the post-processing step [GT18]. Our method overcomes this issue by using the integration-based predictor-corrector method, which is more robust to variations of PV values along banners, as it exploits flow information of the velocity.

A thorough overview of visualization techniques in meteorology can be found in the survey article by Rautenhaus et al. [RBS $\left.{ }^{+} 18\right]$. 



\section{3}

\section{Method}

This chapter states the requirements of a method to facilitate the analysis of PV banners. With these requirements in mind, the developed method is presented which includes the description of the feature extraction, the clustering algorithm to combine similar PV banners and the method to temporarily track the line features.

\subsection{Requirements}

Common practice in meteorology is to visualize 2D slices of potential vorticity [SG16, $\mathrm{SSL}^{+} 03$ ] as shown in Figure 1.1. However, PV banners are three-dimensional structures and thus their visualization in 3D can support mental model building $\left[\mathrm{RBS}^{+} 18\right]$.

In order to learn about the meteorological background and to make the visualization system most useful to our domain scientists, we continuously integrated them into the development process of our visualization system, regularly receiving their feedback and suggestions for improvements. Early on, it became clear that a robust and efficient feature extraction is essential to provide an entry point into the visual exploration and to enable the detailed analysis of PV banners by means of scientific visualizations. Thus, the primary goal of the present work is to identify PV banners from numerical weather prediction data in an automated and robust manner and to visualize them in a way that benefits a subsequent in-depth meteorological analysis of the atmosphere.

Specific requirements have raised the desire for an extraction method in 3D which include:

- R1. An appearance of high PV values can occur due to numerous reasons. The approach should allow us to focus on the relevant PV structures, namely the banners starting from mountain ridges. Other structures can be seen in Figure 1.1 (northwest).

- R2. Our domain scientists are interested in an analysis of the shape of PV banners, including the geometric properties like length and curvature, as well as the vertical distance 
to the surface, the change in altitude and the location of the PV banner's origin on the mountains.

- R3. Further, an analysis of their dynamic behavior is of high interest, including the lifetime of individual banners. How do they deform over time? When do they start and end? Do they become unstable (i.e., break up into pieces)? Which meteorological situations favor the creation of banners and their decease?

- R4. Finally, the system should enable the exploration of the relationship between banners and other meteorological processes such as relative humidity.

Throughout the development process and due to the possibilities provided by the system, our domain scientists raised further questions, digging deeper with us into the spatial and temporal evolution of banners and their interplay with other meteorological processes.

In the following, we describe our extraction algorithm and our visualizations.

\subsection{Method Overview}

Our visualization pipeline consists of three steps: the extraction of potential vorticity banners, their spatial and temporal clustering, and finally the interactive visualization using multiple coordinated views. The steps are illustrated in Figure 3.1. In Section 3.3, we adapt the predictorcorrector method of Banks and Singer [BS94] to extract potential vorticity banners from numerical weather prediction data. Section 3.4 describes the spatial and temporal clustering used to remove duplicates and to establish temporal correspondences. Afterward, Section 3.5 describes the interactive visualizations provided for the visual data analysis, including spatial embeddings in $3 \mathrm{D}$, parallel coordinates, line plots and a flexible node-based editor for interactive filtering, linking and brushing.

\subsection{Extraction of Potential Vorticity Banners}

To capture the geometric shape of potential vorticity banners, we characterize them as extremal lines in the PV scalar field, i.e., as ridge lines or valley lines. Extremal lines are fully captured by the gradient and the Hessian matrix [Ebe12, $\mathrm{KCH}^{+} 18$ ]. For meteorological data, however, Kern et al. $\left[\mathrm{KHS}^{+} 18\right]$ observed that estimating second-order derivatives for the Hessian suffers from amplification of noise and that smooth interpolation-consistent derivatives are difficult to obtain in practice. Banks and Singer [BS94] circumvented this issue in the context of vortex core line extraction by taking advantage of the assumption that the direction of the vorticity vector has little variation compared to the variation of pressure. Since PV is an active tracer, i.e., it does not follow the velocity field exactly, the predictor-corrector approach is a good fit to calculate PV extremal lines. In the following, we describe the iterative algorithm, the seeding, and the termination criterion. 


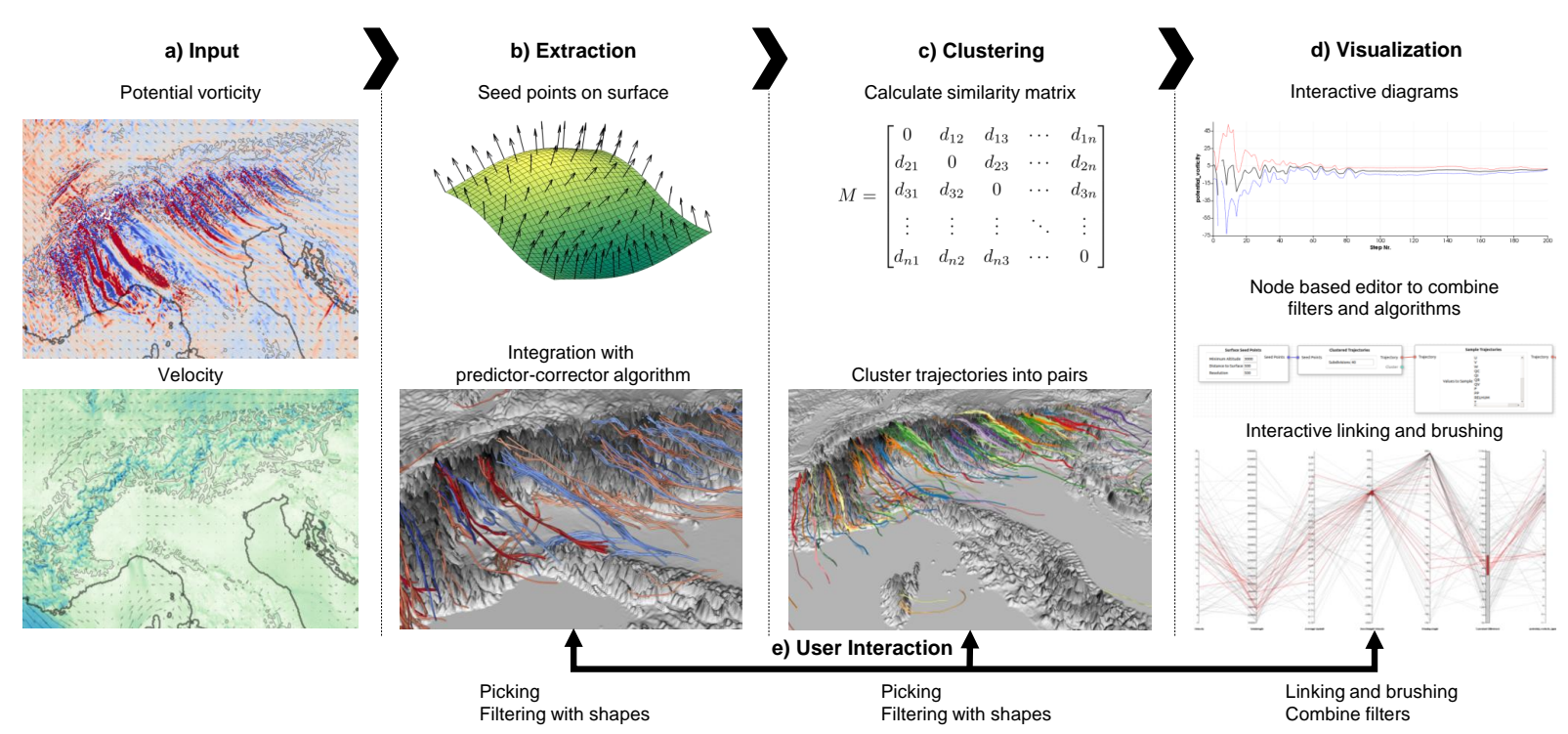

Figure 3.1.: Method overview. (a) The wind velocity and potential vorticity scalar field serve as inputs to our algorithm. (b) The PV banner extraction algorithm distributes seed points along the mountain surface and integrates the banners using a predictor-corrector algorithm. (c) Using a similarity matrix, we cluster similar banners into groups and determine the representatives. (d) Using multiple linked views, a flexible node-based editor and interactive filtering, we can highlight the desired properties of the banners. (e) Users interact with all views by picking as well as linking and brushing.

\subsubsection{Predictor-Corrector Algorithm}

Starting from a given seed point on a potential vorticity banner, the predictor-corrector algorithm generates extremal lines in the potential vorticity field by repeating the following steps:

- Estimate the velocity direction using the fourth-order Runge-Kutta method at the current point in the potential vorticity banner.

- Step in the velocity direction to predict the location of the next line element.

- Construct a plane that is approximately perpendicular to the velocity and find the closest local PV minimum (maximum) using the method of steepest descent (ascent).

- Correct the position by setting it to the potential vorticity minimum (maximum) in the perpendicular plane.

This algorithm differs from the predictor-corrector method of Banks and Singer [BS94] only in the chosen scalar and vector field. We do, however, use an optimization to obtain numerically more robust results. We relax the requirement for an exact perpendicular plane to an, in the vertical axis aligned, approximately parallel plane. This is because the vertical spacing of the data, which is $\approx 20 \mathrm{~m}$ along the surface, is significantly smaller to the horizontal spacing of $2.2 \mathrm{~km}$ in the dataset. Fixing the perpendicular plane to the vertical axis allows for numerically more stable calculations of the gradient and to choose distinct step sizes for the vertical and horizontal direction in the correction step.

The plane is constructed using the velocity $\mathbf{v}=(u, v, w)$ with the three wind components 


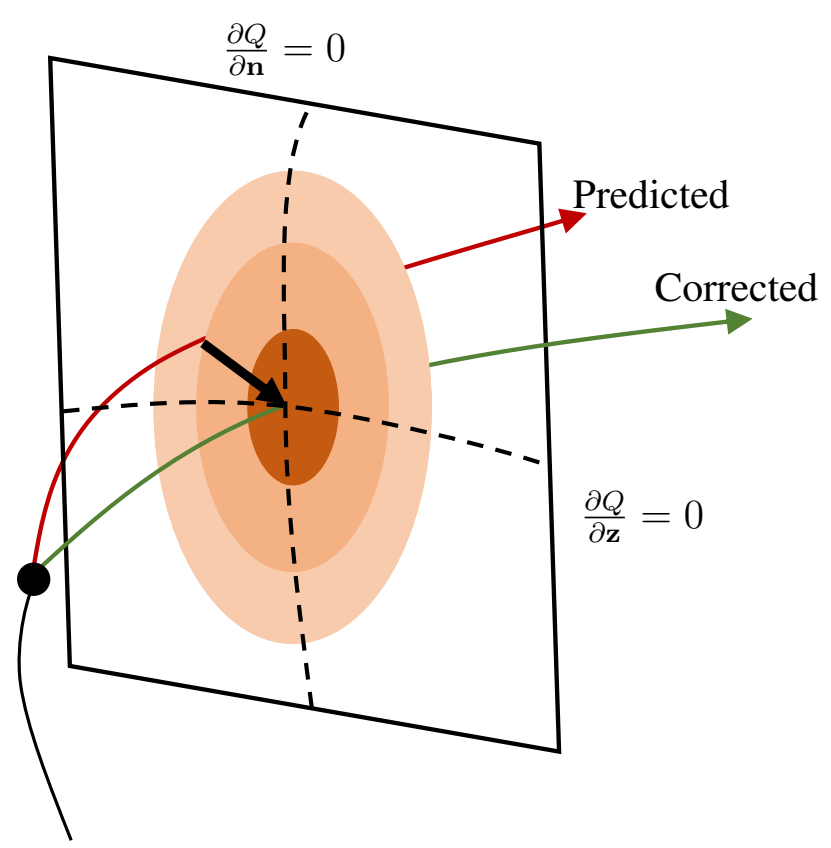

Figure 3.2.: Illustration of the correction step of the predictor-corrector algorithm, which corrects a predicted point by setting it to the potential vorticity minimum (maximum) using the method of steepest descent (ascent). Projecting the gradient on a plane approximately parallel to the velocity allows us to find the extremum at the intersection of the implicit lines where the gradient of each component is equal to 0.

$u, v, w$ defined at grid points at the coordinates $x, y, z$ representing rotated longitude, latitude and surface aligned model levels, respectively. The resulting plane, visualized in Figure 3.2, can be described by following two unit vectors:

$$
\mathbf{n}=\|(-v, u, 0)\|, \quad \mathbf{z}=(0,0,1)
$$

The closest local extrema can be found using the method of steepest descent (ascent). A local minimum (maximum) is given by:

$$
\frac{\partial Q}{\partial \mathbf{n}}=0 \quad \text { and } \quad \frac{\partial Q}{\partial \mathbf{z}}=0
$$

We can derive $\frac{\partial Q}{\partial \mathbf{n}}=0$ by projecting the axis-aligned partial derivatives onto the horizontal unit vector $\mathbf{n}$ :

$$
\frac{\partial Q}{\partial \mathbf{n}}=\mathbf{n} \cdot\left(\frac{\partial Q}{\partial x}, \frac{\partial Q}{\partial y}\right)
$$

Using the method of steepest descent (ascent) we can approximate the closest local minimum (maximum) by repeatedly correcting the actual predicted point on the plane into the negative (positive) direction of the projected gradient.

The search for the minimum (maximum) in the plane is terminated when the magnitude of the gradient is below a threshold, a maximum number of steps is reached, the change in PV is below a threshold or if the angle between the predicted and corrected vector is above a specific threshold to prevent the swapping between two neighboring banners. 


\subsubsection{Seed Points}

The integration-based method of the predictor-corrector algorithm requires the placement of seed points. In the atmosphere, line-like structures of potential vorticity can develop potentially at any point. However, potential vorticity banners originate from orographic peaks and massifs, see Section 2.2 and Figure 2.1 (a). This enables us to take advantage of this property by carefully placing seed points close to the terrain surface. Aside from fulfilling requirement R1, i.e., discarding all PV structures that are not PV banners, this allows us to establish the connection between the PV banner and its origin at the mountain (R2).

\subsubsection{Banner Termination \& Filtering}

Much like the pressure in a vortex core line, the PV value varies along a PV banner, due to meandering and dissipation, making the threshold-based termination responsive to noise in the data. Thus, we terminate the iterative tracing of a PV banner only when the PV value remains below a user-defined threshold for a certain distance. We defined the distance to be $8.8 \mathrm{~km}$ which is approximately 2 horizontal grid cells and the absolute PV threshold to be smaller than 1.0 PVU. The algorithm can extract very short line structures or banners, which can be filtered by the absolute potential vorticity, the accumulated potential vorticity, or a minimal geographic banner length.

\subsection{Clustering of Potential Vorticity Banners}

The predictor-correct method can lead multiple seeds onto the same potential vorticity banner since an overabundance of seed points produces a multitude of nearly coincident banner lines. We eliminate the duplicates by taking advantage of the fact that the predictor-corrector method is convergent to the PV core line. Using a distance measure we can find very close lines and classify them as one general PV banner, choosing a representative line. This is required to enable a quantitative analysis of the banners (R2), e.g., to compute the average length and the number of banners.

\subsubsection{Distance Metric}

The distance measure is required to be positive-definitive, symmetric and it needs to have a low sensitivity to different line lengths. A survey of different line similarity measures complying to these requirements was given by Oeltze et al. $\left[\mathrm{OLK}^{+} 14\right]$. They concluded that the reduced mean of closest point distances (rMCPD)

$$
\begin{aligned}
d_{H}\left(s_{i}, s_{j}\right) & =\min \left(d_{h}\left(s_{i}, s_{j}\right), d_{h}\left(s_{j}, s_{i}\right)\right) \\
\text { with } d_{h}\left(s_{i}, s_{j}\right) & =\operatorname{mean}_{p_{l} \in s_{i}} \min _{p_{k} \in s_{j}}\left\|p_{k}-p_{l}\right\|
\end{aligned}
$$

which is an adaptation of the MCPD [CGG05], is especially robust for different line lengths compared with the Hausdorff-Distance [RT12] and the regular MCPD. The sensitivity of rMCPD 


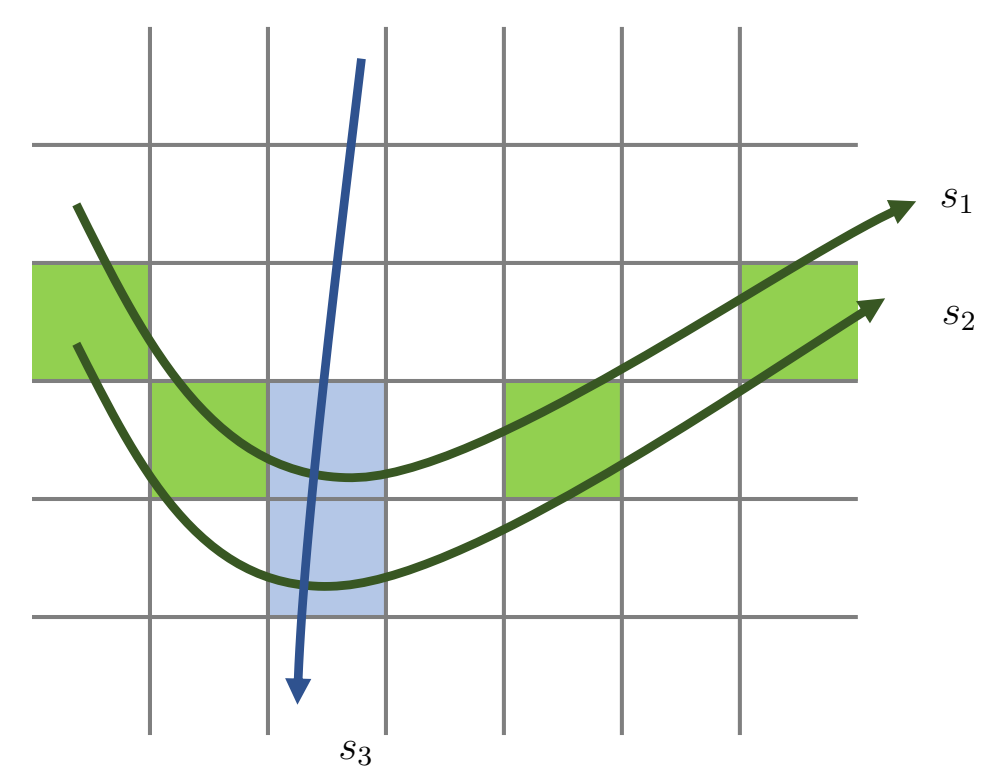

Figure 3.3.: Cluster algorithm using AHC. The grid represents a spatial data structure. Lines that share a grid cell are compared with each other, e.g., the blue or green shaded cells highlight overlapping line elements in the spatial grid. $s_{1}$ and $s_{2}$ have a high similarity measure and are clustered together. None of the lines have a high similarity with $s_{3}$ and are therefore clustered independently.

is reduced by replacing the outer mean of MCPD by a minimum computation in Equation (3.4). For banners with different signs we set the distance to infinity to avoid clustering of inherently different banners.

\subsubsection{Spatial Clustering}

To group PV banners and to select representative lines, we use the Agglomerative Hierarchical Clustering (AHC) $\left[\mathrm{OLK}^{+} 14\right]$. The algorithm requires a squared symmetric distance matrix $M$ as a measure of cluster proximity, where $M$ contains the pairwise inter-line proximity of all PV banners to each other. We construct the matrix $M$ using the rMCPD distance metric. AHC starts with each line being a cluster and then repeatedly merges the two closest clusters until no cluster is within a user-defined distance threshold. This termination rule has the advantage, compared with other clustering methods such as K-means, that the number of clusters does not have to be known beforehand.

AHC has a high time complexity to compute the matrix $M$, which requires that all lines need to be compared to each other using the distance metric. To accelerate the computation, we construct a spatial data structure to only compare potentially close enough clusters, (i.e., below the distance threshold) with each other, see Figure 3.3. The banners are finally represented by the cluster member with the highest proximity to the other members. Figure 3.4 gives an example of the obtained clustering, visualizing each cluster with a different color. Details on the performance of the algorithm can be found later in Section 5.3. 


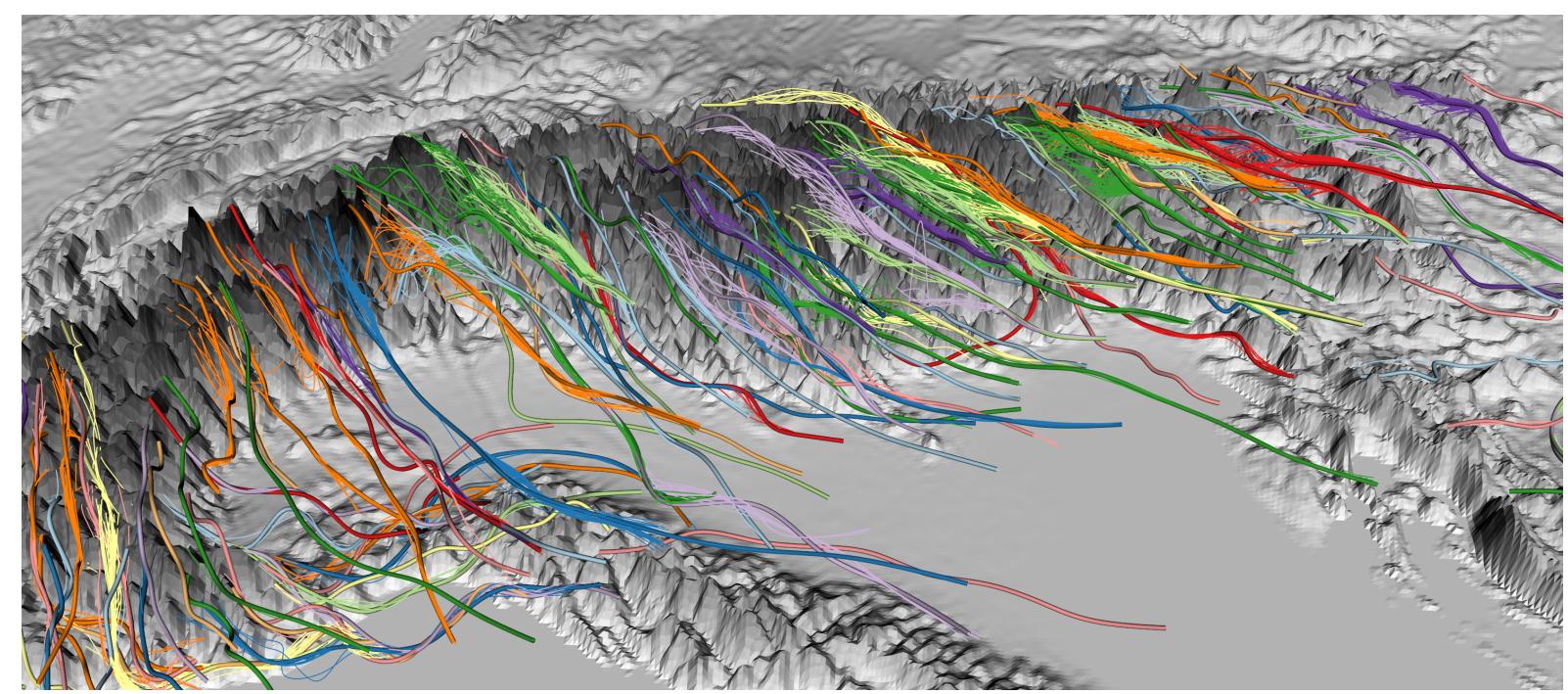

Figure 3.4.: The PV banners are colored based on their cluster membership. This enables lines to be categorized together that are integrated from different seed points but converged into the same banner. We apply the AHC to group banners and select representative lines.

\subsubsection{Temporal Clustering}

To enable the observation of PV banners over time (R3) it is necessary to follow specific banners, e.g., to measure their lifetime. Further, this allows us to raise additional analysis question, e.g., how long does a banner stay stable? How does its curvature, length or vertical extent behave over time?

We implemented this requirement by clustering over time. As input we take the cluster representatives found with AHC of the time steps $t_{i}$ and $t_{i+1}$. By connecting each representative in $t_{i}$ with the closest representative in $t_{i+1}$ we create a many to 0 relationship through the time steps as visualized in Figure 3.5 (a). If a representative line of $t_{i}$ has no successor the line is seen as dissolved by the algorithm. If, however, several representatives in $t_{i}$ connect to the same banner in $t_{i+1}$ we count them as merged together. The same way we can identify newly arising banners if no line in $t_{i}$ is present for a representative in $t_{i+1}$. Figure 3.5 (b) shows an example of the algorithm where a selection of banners was observed over time for 24 time steps with a delta time of 10 minutes (4 hours in total). In principle, a number of other feature tracking algorithms are available that could likewise be used, see Saikia and Weinkauf [SW17]. In order to increase the temporal coherence, we employ a Lagrangian smoothing of the PV field, i.e., the PV value at a certain grid point is calculated by convolving the PV scalar field along a pathline, weighted by a Gaussian filter function, similar to a line integral convolution [CL93]. The supplemental material contains a video sequence, comparing the temporal clustering with and without Lagrangian smoothing. 


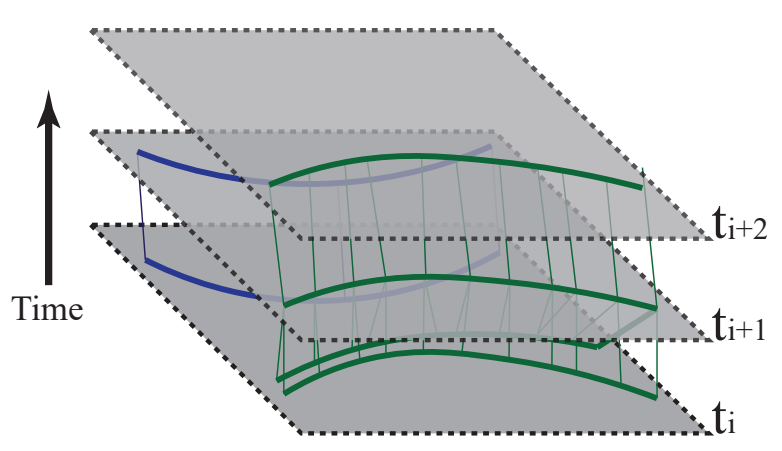

(a)

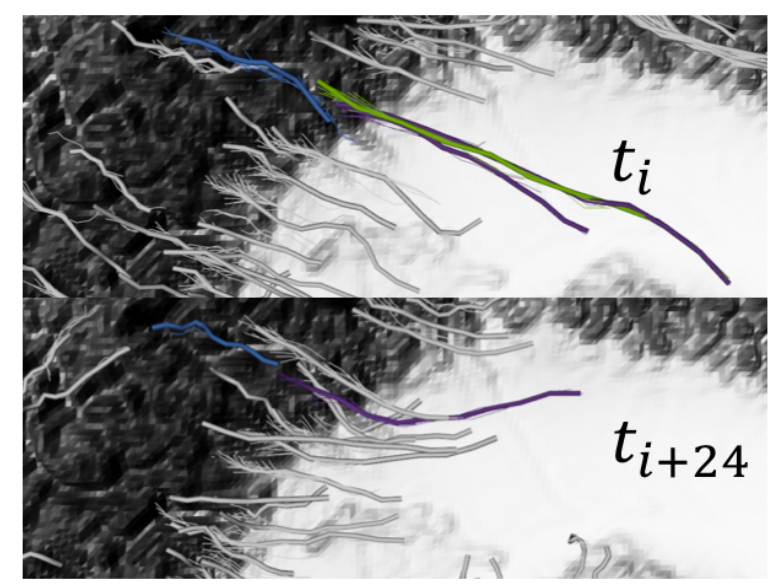

(b)

Figure 3.5.: (a) In order to observe the temporal evolution of PV banners, we combine the closest lines between time step $t_{i}$ and $t_{i+1}$ in a temporal structure. (b) Example of the temporal banner tracking, when following a selection of PV banners 4 hours in time (24 time steps). The selection stays stable for the banners. The green banner dissolved over time.

\subsection{Visualization}

Given the PV banners in 3D, we implemented the pathline attributes of Shi et al. [STHW07] to compute a number of derived properties, including line length, accumulated curvature, and life time. Some properties are locally computed per line vertex, others summarize the line behavior globally, such as the average direction, curvature, or the surrounding relative humidity. Our visualization system ties together three interactive views, which include a 3D geospatial view, multi-variate information visualizations, and a time-series view. Each view gives a different perspective on to the data and allows for real-time exploration. Furthermore, each view is linked so that selections and interactions in one view simultaneously affect the other as visualized in Figure 3.7 (a) and 3.7 (b). In the following, we discuss the three views and our node-based visualization and filtering system in more detail.

\subsubsection{Geospatial View}

In the 3D view, $\mathrm{PV}$ banners are visualized as colored tubes with the topography being rendered in the background to provide a geospatial context. In order to enable the analysis of correlations among line attributes (R2, see requirements in Section 3.1) and with meteorological attributes (R4), properties can be mapped to the radius, color, and opacity of the tubes. Embedded into the 3D space, the viewer obtains an impression of the general PV banner shape and their alignment with wind vectors.

Displaying the full set of banners may result in visual clutter. We, therefore, provide two approaches to reduce the amount of data. First, the user can interactively place regions-of-interest to select individual banners, as shown in Figure 3.7 (c). The banners are then highlighted, whereas the remaining lines are desaturated, providing focus and context, as done in Figure 3.5 (b). 


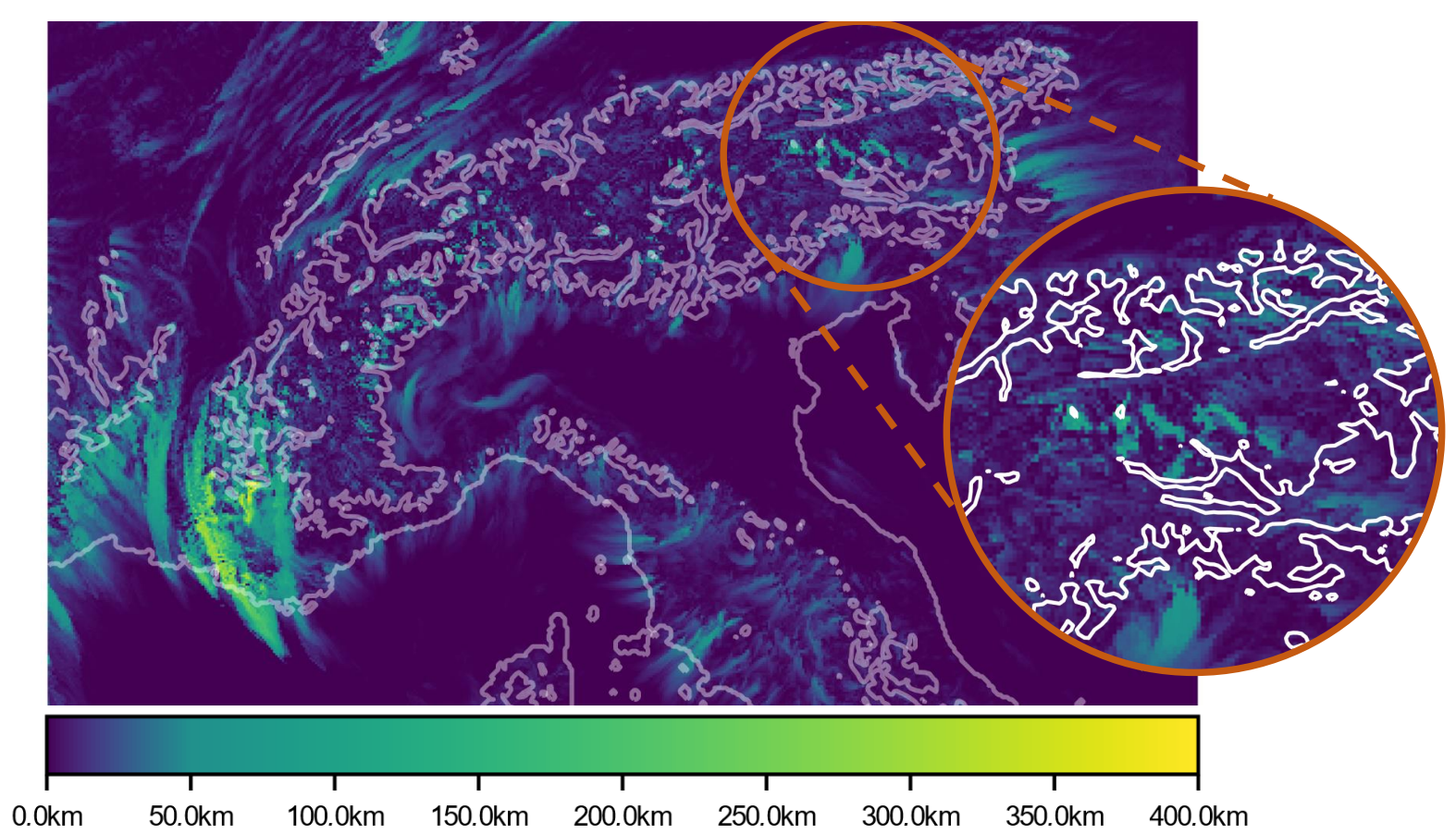

Figure 3.6.: Time-dependent projection of the $P V$ banners length onto their seed points. Enables perception of geographical important banner seed points. The long banners in the east are part of a primary $P V$ banner in the scale of the whole Alps. Several high peaks (magnified) show an increased banner length compared to their surroundings. Time period: 29 January 2010 00:00 to 08:00 UTC.

Second, since all relevant PV banners were released from the mountains (R1), line properties can be directly mapped to the seed point. This way, a scalar field is obtained that is directly embedded on the mountain surface, which is a 2D manifold in the 3D space. Figure 3.6 shows a time-dependent projection of the average banner length onto the surface.

To study correlations with meteorological attributes $(\mathbf{R 4})$, such as the updraft wind component, relative humidity, or potential vorticity itself, a 2D plane can be placed in the scene. For a selected PV banner, the upright-oriented plane can be moved interactively along the line. With this, atmospheric parameters can be directly visualized in the vicinity of the banner. The plane can be aligned perpendicular to the line, tangential to it or in the horizontal layer. Furthermore, glyphs can be placed on the plane for instance to show the direction of three-dimensional vectors, e.g., velocity, vorticity, gradients of scalar values, as done in Figure 2.2.

\subsubsection{Multi-Variate View}

In order to explore the multi-variate attributes $(\mathbf{R 2}, \mathbf{R 4})$, common information visualization techniques are used to discover relevant correlations, interesting feature combinations, or general properties of the data. Our application supports 2D scatter plots, parallel coordinates and histograms, as shown for instance in Figure 3.7 (a) or Figure 5.2. We employ linking and brushing, i.e., users can select certain properties in one view which are then propagated onto all linked views by an appropriate focus and context switch. This combination of views makes it possible 


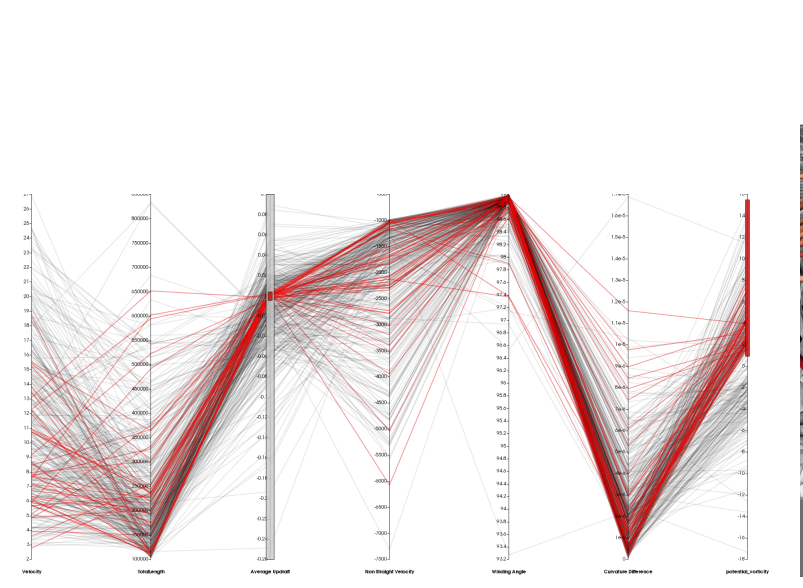

(a)

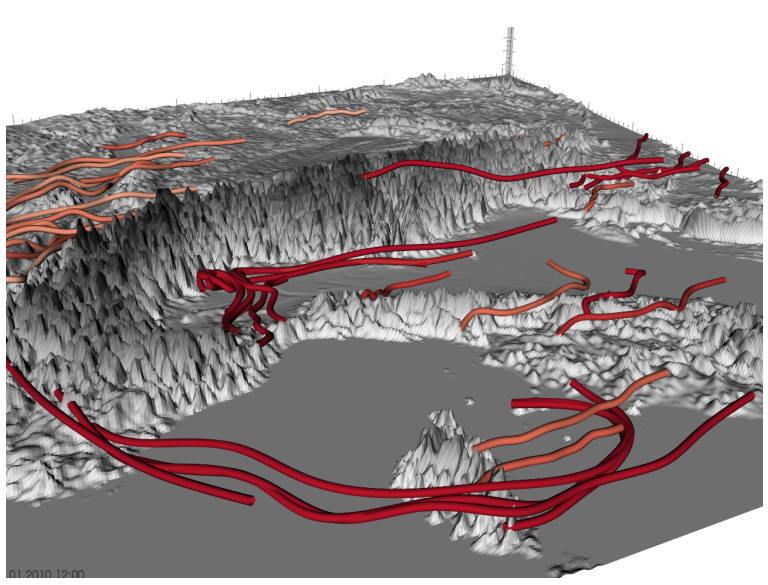

(b)

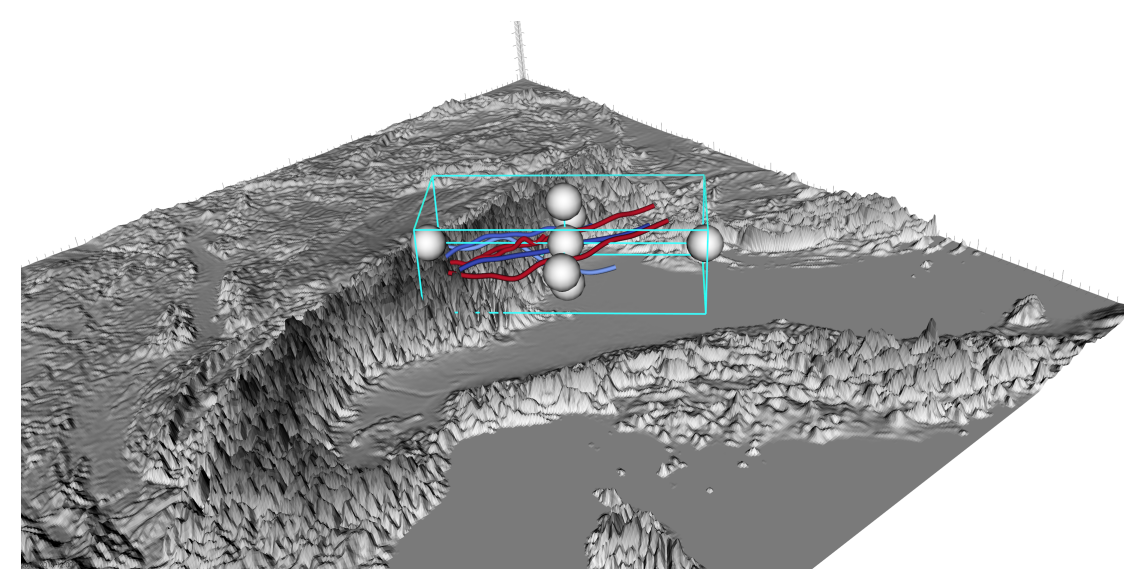

(c)

Figure 3.7.: Example of linked views. (a) Parallel Coordinates Diagram. Selection of positive PV banners with low updraft. Selection automatically updates all other views. (b) Updated view according to selection in (a). (c) Limit selection by placing shapes within the scene. E.g., box that filters banners outside of it.

to analyze a wide variety of scalar quantities.

\subsubsection{Time-Series View}

While the previously presented visualization techniques are effective for comparing trends between variables as well as spatial differences throughout the domain, they are not effective for the visualization of trends over time (R3). For this, we implemented a separate time-series view that displays the temporal properties of selected banners in interactive line plots, as shown later in Figure 5.3. We rely on the temporal clustering from Section 3.4.3 to establish the temporal correspondence, which in turn allows us to infer information about a banner's life cycle. The different lines through time can be interactively compared or accumulated as done in Figure 3.6. 


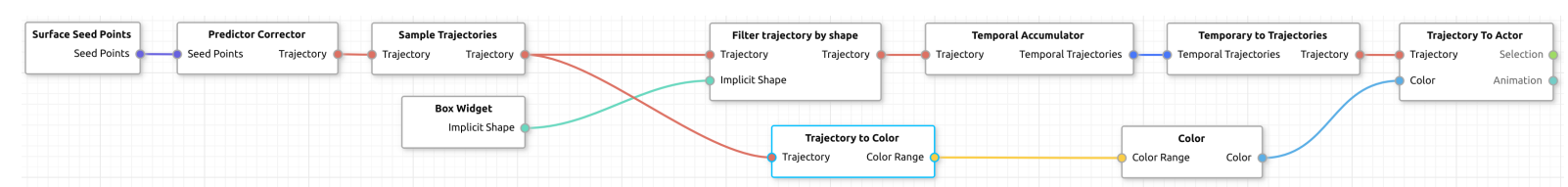

Figure 3.8.: Data flow programming implementation. Enables a high degree of flexibility by defining combining operators through connections while hiding the underlying algorithmic complexity. This specific example samples seed points along the surface, integrates the banners using the predictor-corrector algorithm, filters the banners that are within a user defined box and shows the temporal accumulation of them in the view using the depicted color maps.

\subsubsection{Node-based Editor}

As suggested by Treinish [Tre02] visualization methods in meteorology require a very generic approach. The demand for a flexible and complex composition of different algorithms, filters and visualization techniques led us to incorporate a data flow paradigm that internally represents the data processing pipeline as a directed graph. Further details on the design choices are discussed in Section 5.2.

To allow this high degree of flexibility while hiding the complexity of the underlying algorithms from the user a data flow programming paradigm [JHM04] was implemented. Data flow programming allows the definition of a program as a directed graph where the data flows between operations. Explicitly defined inputs and outputs connect operations that are executed as soon as all of their inputs become valid.

Each algorithm, filter or visualization technique is represented as a set of nodes with input and/or output ports in them. These nodes can either be sources, sinks or processing blocks and are connected by directed edges. This approach enables the visualization of the data flow and offers the domain scientist with a simplified interface to the underlying complex algorithms.

During this project, a multitude of different types of nodes (See Table A.1) were developed which enable a domain scientist to compose complex graphs that define the data flow and the visualizations of the application. This simplified interface can provide rapid prototyping and implementation of desired results.

The resulting user interface is shown in Figure 3.8, allowing the creation of seeding structures, visualization methods, and visualization parameters.

Further details on the implementation can be found in Section 4.3. 



\section{4}

\section{Implementation}

This chapter is structured into three parts. Firstly, we introduce into the data used to extract the PV banners. This data has specific properties that need to be handled properly, which leads us to the introduction of the mathematical and algorithmic basics of the COSMO numerical weather data. Finally, we go into detail with the software implementation by describing the implemented software architecture which was developed to enable a flexible development environment by abstracting the problem into sub-layers.

\subsection{Data}

This work utilizes data from the COSMO (Consortium for Small-scale Modeling weather and climate model), which is a nonhydrostatic limited-area model that solves the fully compressible governing equations using finite difference methods on a structured grid [SDS ${ }^{+} 03$ ]. The data is computed using a refactored version of COSMO capable of using GPU accelerators $\left[\mathrm{FOL}^{+} 14\right]$ that allows the refinement of the model resolution to the kilometer scale on continental scales. The advantage of high-resolution km-scale models is a better representation of underlying orography and the associated flow, and it allows the explicit representation of convection (i.e., thunderstorms and rain showers, vertical circulation - updrafts and downdrafts) that conventional weather and climate models are not suited to resolve without parameterization [BSS15, $\left.\mathrm{LFL}^{+} 16\right]$.

The model is discretized on a rotated latitude-longitude grid using terrain-following altitude coordinates. The configuration has a convection-resolving horizontal grid spacing of $2.2 \mathrm{~km}$ and $1536 \times 1536 \times 60$ grid points with a time step of 20 seconds. The vertical direction is discretized using 60 stretched model levels from the surface to the model top at $23.5 \mathrm{~km}$. The respective layer thickness widens from $20 \mathrm{~m}$ at the surface to $1.2 \mathrm{~km}$ near the model top. The necessary initial and boundary condition for the model are derived from the European Centre for MediumRange Weather Forecasts (ECMWF) ERA-Interim reanalysis [DUS $\left.{ }^{+} 11\right]$ and are updated every 


\section{Implementation}

$6 \mathrm{~h}$. We followed the model setup defined in Leutwyler et al. [LLB $\left.{ }^{+} 17\right]$.

From the simulation domain, a subset of $500 \times 500 \times 60$ grid cells over the Swiss Alps was extracted to analyze the potential vorticity banners over the Alps. Figure 4.5 (a) and (b) compare the original grid extent with the extracted subset. Nikolina Ban, from IAC, provided two weeklong cases starting at April 10, 2000 and January 27, 2010, and output the data every 10 minutes. In total, the numerical simulation data accumulated to $14 \mathrm{~TB}$ of raw data. The subset of the data including additional derived values accumulated to additional 3 TB of optional data.

\subsection{Details to numerical data}

PV is a derived scalar field and calculated according to Equation 2.3. The output generated by COSMO serves as the data foundation for this project and the required variables to calculate PV are listed in Table 4.1. All the variables, with the exception of the constant ones, are timedependent and are each represented by individual files based on their time and content. The data is given in NetCDF format with the convention version CF-1. $4^{1}$ with a fixed bit offset of $32 \mathrm{bit}$ per variable.

For a variable with the dimensions $1542 \times 1542 \times 60$ this results in a size per variable of approximately $544 \mathrm{MB}$ per file. To extract PV banners we need at all times the variables $U, V, W, P, T$ and optionally RELHUM in memory for each time step which results in $6 \times 544 \mathrm{MB} \approx 3.3 \mathrm{~GB}$ per time step. To enable fast processing, which is a requirement for a responsive interaction with the users, it is beneficial to have all these files loaded in memory. To reduce the memory footprint of the application a user-defined subset in the horizontal space can optionally be defined. This results in significantly faster loading times of the application and decreased requirements on disk space for preprocessed files or the application's caching system. For all visualizations in this project the subset $[700: 1200] \times[450: 900] \times 60$ which covers the European Alpine region was chosen as visualized in Figure 4.1 and Figure 4.5.

The data generated by COSMO generates data in a so-called surface aligned grid. This data structure is frequently used in numerical weather simulation data because it samples the atmosphere in varying resolutions based on the expected variance. For example, along the surface where a high variance is expected the data is sampled in higher vertical resolution (from $20 \mathrm{~m}$ spacing in the vertical) compared with the model's higher altitude levels where the vertical spacing is sampled with lower resolution $(1.2 \mathrm{~km}$ spacing). Additionally to this data structure, the coordinate system of the COSMO data is a rotated version of the geographical coordinate system. This surface aligned grid and the rotated coordinate system require a careful conversion to enable a visualization in 3D which affects the following parts of the implementation:

- Sampling of values in the three-dimensional grid cells.

- Indexing in surface aligned grid.

- Rotated Longitude/Latitude Grid conversions.

- Calculation of distances on Spherical Earth.

${ }^{1}$ http://www.cfconventions.org/ 


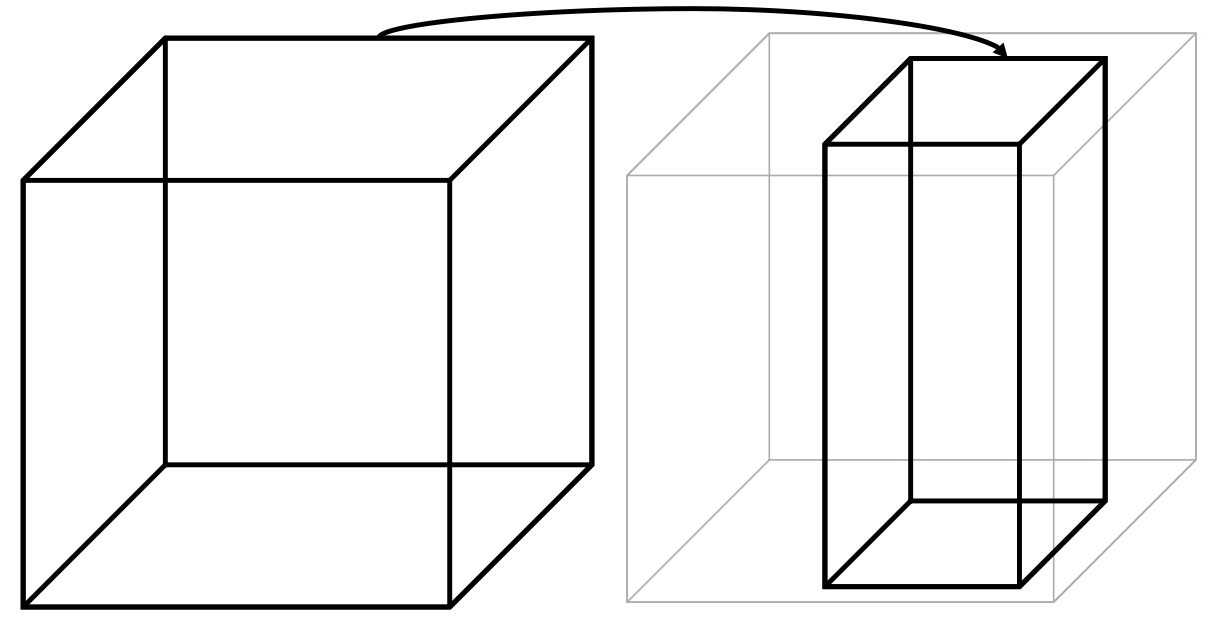

Figure 4.1.: Schematic overview of subset extraction. In the three dimensional grid a horizontal subset can optionally be extracted to increase loading times and optimize memory footprint.

- Finite Differences in surface aligned grid.

- Converting from Meter above sea level to index in grid cells.

- Interpolation of values between grid cells.

\subsubsection{Cell Sampling}

The data is sampled into cells and the exact sampling position depends on the vertical dimensions. As listed in Table 4.1 the vertical dimensions either consists of the values 1, 60, 61 which stands for two-dimensional data, three-dimensional data sampled in the cell center and threedimensional data sampled at the cell boundaries (Half-level-sampling), respectively.

An example of a two-dimensional datasets is the variable $H S U R F$ representing the surface altitude. This convenient case does not need to handle specific sampling strategies for the vertical component and the values are mapped to each cell's center which is also known as a staggered grid.

The three-dimensional variables require more complex handling to be sampled. All the variables with exactly $l=60$ levels, as provided by the COSMO simulations, are sampled on the cell's center. The vertical location of the cell's center needs to be derived from their vertical cell boundary locations, so-called half-levels. These half-levels are represented by variables with $h h l=l+1=61$ levels and are schematically visualized in Figure 4.2. An example to derive the cell center using the altitude variable $H H L$ is given by:

$$
h_{x, y, l}=\frac{h h l_{x, y, l+1}-h h l_{x, y, l}}{2}
$$




\begin{tabular}{lllll}
\hline File & Variable Name & Description & Vert. dims. & Unit \\
\hline Constants & HHL & Half Level Altitude in Meter & 61 & $\mathrm{~m}$ \\
Constants & HSURF & Surface Altitude & 1 & $\mathrm{~m}$ \\
Winds & U & Grid eastward wind & 60 & $\frac{\mathrm{m}}{\mathrm{s}}$ \\
Winds & V & Grid westward wind & 60 & $\frac{\mathrm{m}}{\mathrm{s}}$ \\
Winds & W & Grid vertical wind & 61 & $\frac{\mathrm{m}}{\mathrm{s}}$ \\
Other & P & Air pressure & 60 & $\mathrm{~Pa}$ \\
Other & T & Air Temperature & 60 & $\mathrm{~K}$ \\
Other & RELHUM & Relative Humidity & 60 & $\%$ \\
Clouds & QV & Specific Humidity & 60 & $\frac{\mathrm{kg}}{\mathrm{kg}}$ (fraction) \\
Clouds & QI & Specific Cloud Ice Content & 60 & $\frac{\mathrm{kg}}{\mathrm{kg}}$ (fraction) \\
Clouds & QC & Specific Cloud Liquid Water Content & 60 & $\frac{\mathrm{kg}}{\mathrm{kg}}$ (fraction) \\
Clouds & QR & Specific Rain Content & 60 & $\frac{\mathrm{kg}}{\mathrm{kg}}$ (fraction) \\
\hline
\end{tabular}

Table 4.1.: Listing of scalar values required to extract $P V$ banners and generate the presented results.

where $h_{l}$ refers to the altitude of a level's center and $h h l_{x, y, z}$ refers to a the altitude of the cell's vertical boundaries, i.e., half-levels.

\subsubsection{Cell Indexing in Surface Aligned Grid}

The COSMO simulation persists the data in a surface aligned grid which requires great care while indexing. Given a variable $V$ a value at a three-dimensional position can be accessed by the index $V_{x, y, z}$ where $x, y, z$ refer to rlon, rlat, $h h l$ as described in Figure 4.4. A property of this dataset is that the maximum altitude is accessed with the altitude index $z=0$ and the cell at the earth's surface is accessed using $z=60$.

The surface aligned sampling grid has advantages in the numerical computation of the boundaries as well as it compresses the data by enabling a higher sampling along the surface where more changes happen as in higher altitudes. In the computation of the values, a careful interpolation is necessary to get the right cell at each altitude level.

In the horizontal slices, the sampling distance between a cell is regular, i.e., all cells centers have a fixed distance to their neighbors. In contrast, the vertical spacing of the cell depends on the surface altitude as the data is sampled on a surface aligned grid as shown in Figure 4.3. The data index 0 refers to the model's maximum altitude boundary in the atmosphere whereas the data index 60 refers to the model's surface altitude. In between the data is sampled in irregular distances which are originally based on pressure levels. 


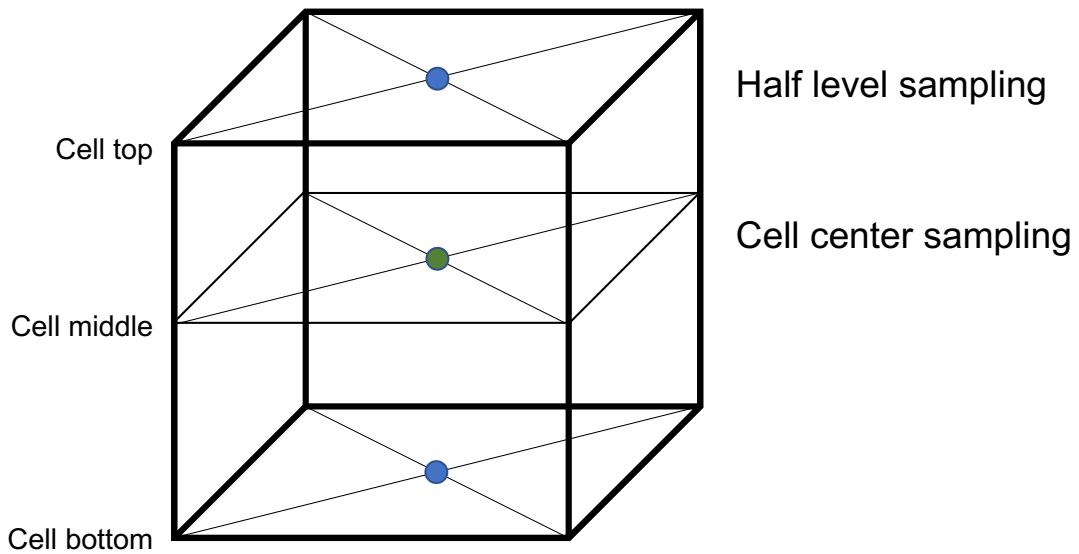

Figure 4.2.: Schematic overview of cell sampling onto half levels or the cell's center. Depending on the resolution in the vertical the data is sampled in different locations.

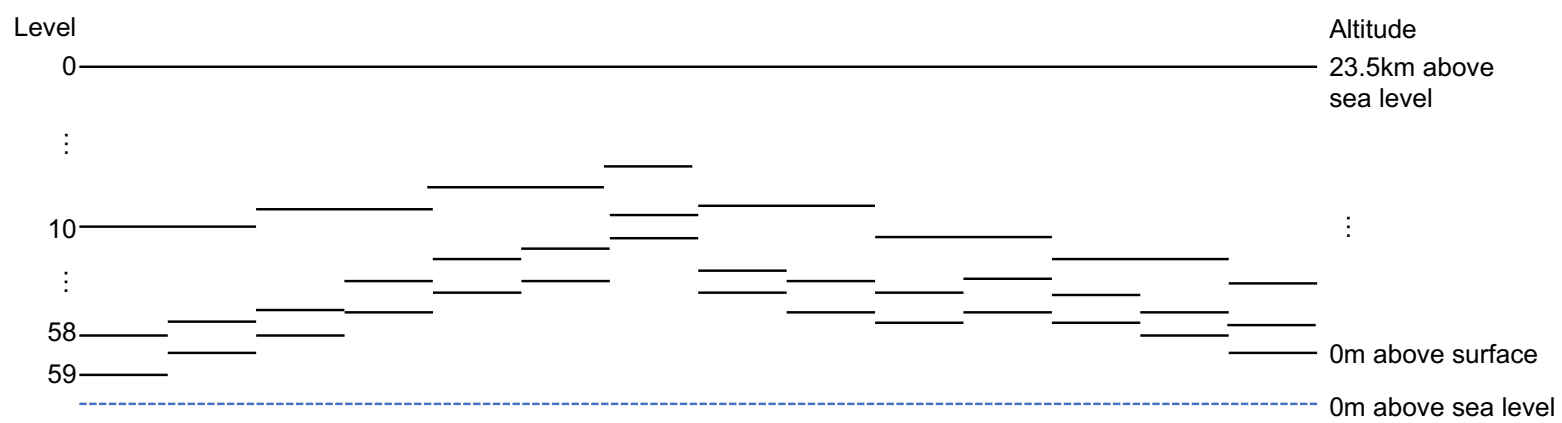

Figure 4.3.: Surface aligned grid where the data is sampled in higher resolutions along the surface which. This data structure optimizes the storage requirements to persist the data. The visualization emphasizes the irregularity of the levels altitudes shown as lines

This affects the following parts of the application:

- Gradient computation

- Integration of the velocity

\subsubsection{Rotated Longitude/Latitude Grid}

The horizontal grid is a regular grid in a rotated latitude/longitude coordinate system [FOL $\left.{ }^{+} 14\right]$. In the provided datasets the north pole of the rotated grid is transformed to the position $\left(43.0^{\circ},-170.0^{\circ}\right)$ in the geographical latitude/longitude coordinate system. This has the advantage that the coordinate $\left(0^{\circ}, 0^{\circ}\right)$ in the rotated coordinate system lays over the Alpine region as opposed to the north pole as in the geographical coordinate system.

To convert between latitude (lat) / longitude (lon) and rotated latitude (rlat) / rotated longitude 


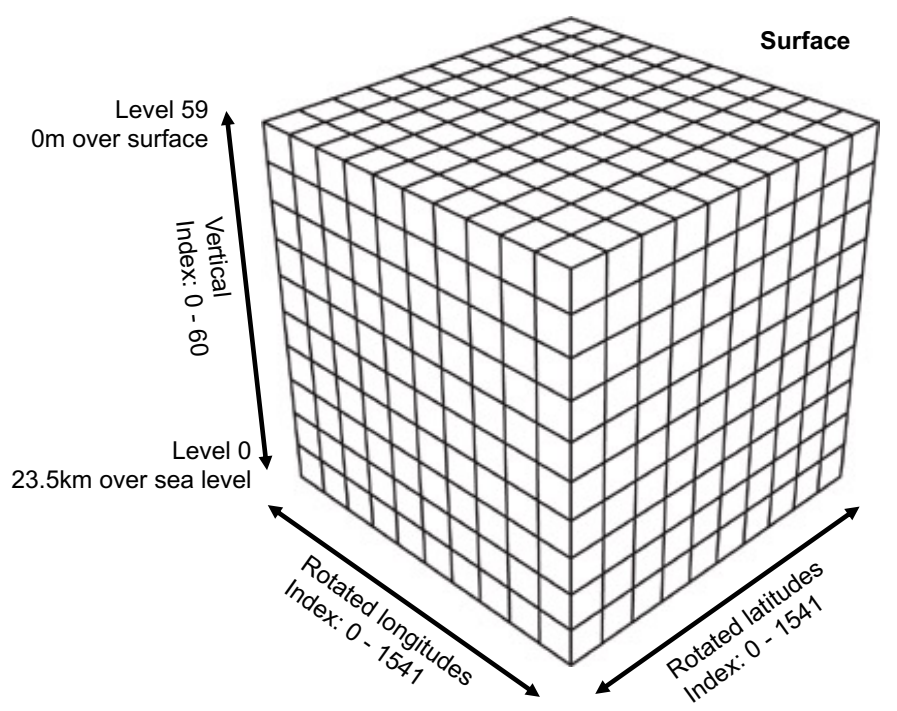

Figure 4.4.: Indexing in surface aligned grid. The vertical index $z=0$ refers to the highest level in the altitude.

(rlon) we use Algorithm 1 as given by the Lagrangian Analysis Tool (LAGRANTO) [MPW13, SW15] which takes as input the rotated coordinates in radians and converts them to geographical coordinates.

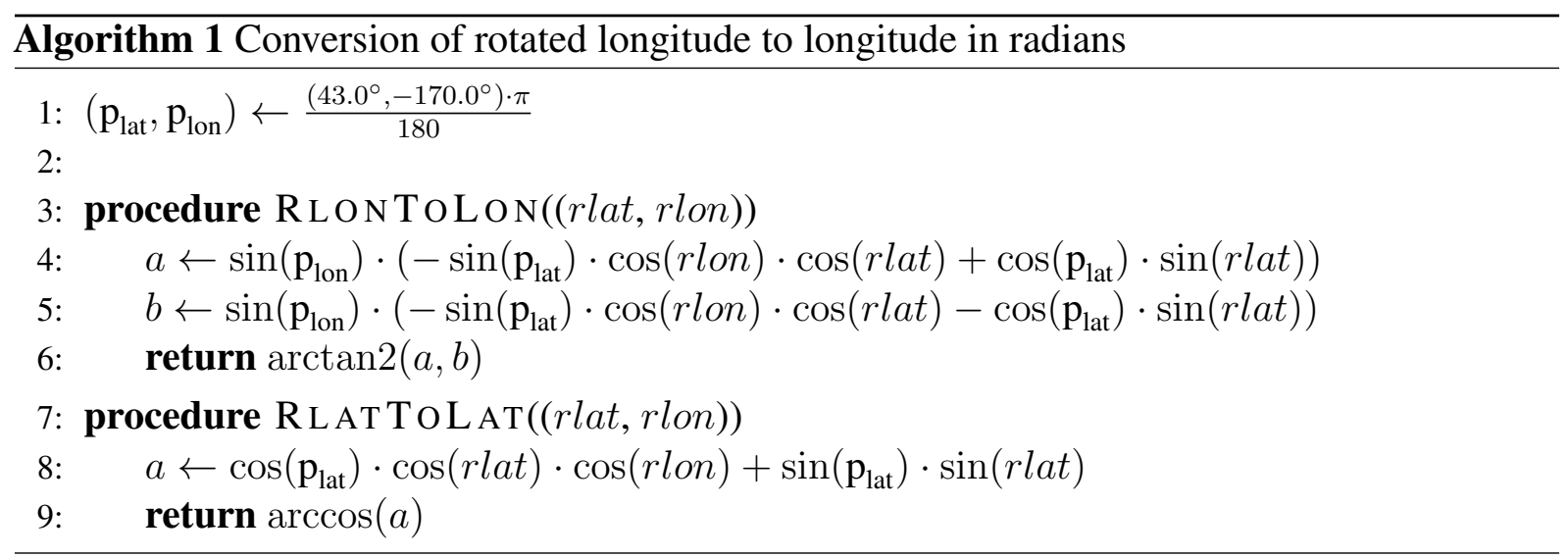

\subsubsection{Calculation of distances in Spherical Earth}

Converting metrical units, for instance, the wind speed which is defined in the unit $\mathrm{ms}^{-1}$, into distances along the spherical Earth and vice versa requires a consistent conversion between the different units.

The horizontal grid is defined in rotated latitude/longitude space. There exist different methods to convert geographical coordinates into meter. They differ about the assumptions of the shape of the Earth. 


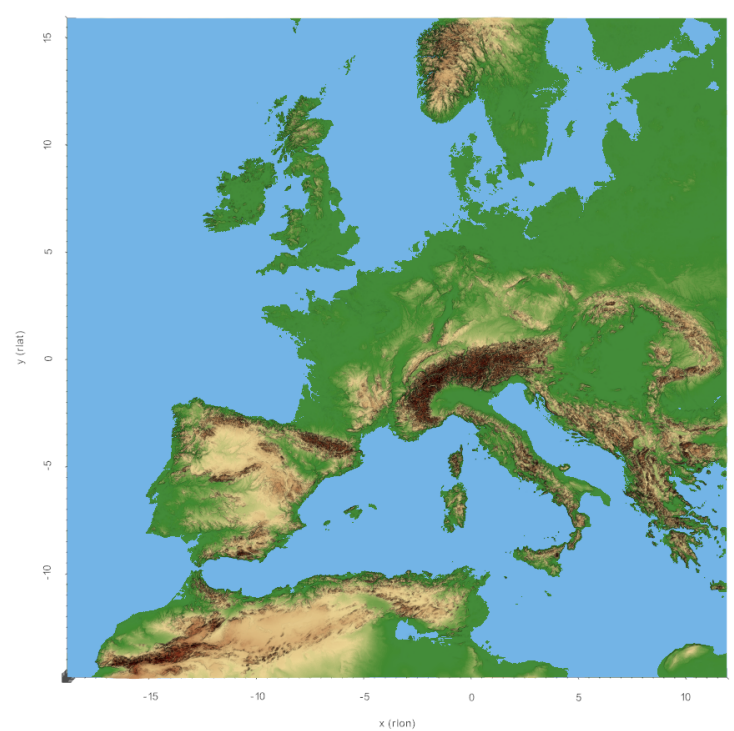

(a)

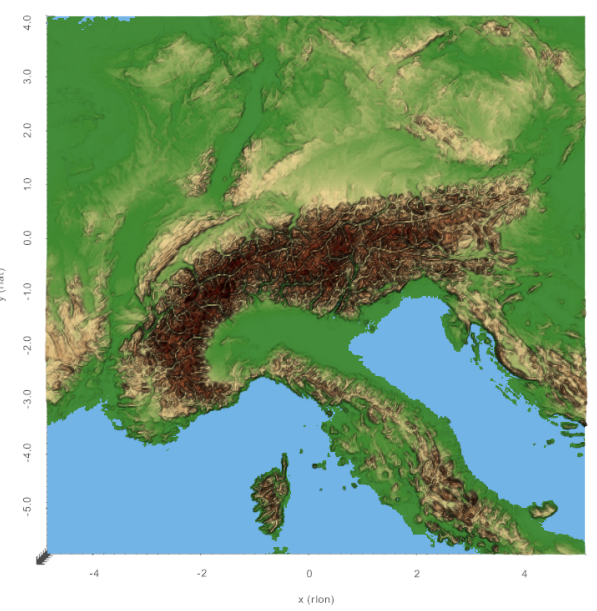

(b)

Figure 4.5.: Comparison of cropped subregion used to present the results of this work with the original datasets extent. (a) Full extent of provided data in rotated longitude and latitude coordinate system. (b) Cropped subregion of simulation data over the Swiss Alps used in project.

As in the Lagrangian analysis tool (LAGRANTO) [MPW13, SW15] we assume a spherical earth. Since the Earth is not spherical that result can be off by several tenths of a percent ${ }^{2}$.

To estimate the length of a degree in meter on a spherical Earth we use Equation 4.2 where Earth's average meridional radius $M_{r}$ is $6^{\prime} 367^{\prime} 499 \mathrm{~m}$.

$$
\frac{\pi}{180} \cdot M_{r}
$$

While the distance between two latitudinal points is constant over the globe, the longitudinal distance decreases towards the poles $\left[\mathrm{KHS}^{+} 18\right]$. This is also the case in the rotated coordinate system.

With the assumption of the spherical globe we approximate the distance in the rlon dimension by scaling the equatorial distance from Equation 4.2 by the cosine of the rotated latitude rlat as in Equation 4.3.

$$
\frac{\pi}{180} \cdot M_{r} \cos \left(\frac{\pi}{180} \cdot \text { lat }\right)
$$

\subsubsection{Finite Differences in Surface Aligned Grid}

The calculation of partial derivatives in $x$ or $y$ direction (i.e., rlon or rlat), needs to incorporate the altitude changes between the neighboring cells.

\footnotetext{
${ }^{2}$ https://en.wikipedia.org/wiki/Geographic_coordinate_system\#Expressing_ latitude_and_longitude_as_linear_units
} 


\section{Implementation}

Assuming we take the partial derivative in $x$ direction of a cell with the scalar variable $V_{r}$ we compute in a regular grid the partial derivative using finite differences and second-order accuracy using the coefficients as in Equation 4.4 where $s$ is the spacing between the neighboring cells in meter.

$$
\frac{\partial V_{r}}{\partial x} \approx \frac{1}{s}\left(-V_{x-1, y, z}+V_{x+1, y, z}\right)
$$

In comparison to this Equation 4.5 shows the approximation in a surface aligned grid $V_{\mathrm{HHL}}$ when the neighboring levels are not of the same height where we need to incorporate this change by adding the vertical partial difference where $h_{v}$ refers to the vertical altitude difference and $h_{h}$ refers to the horizontal altitude difference. When taking the partial derivative with respect to the vertical $z$ axis we need to incorporate the flipped indexing where the higher cell is an index below the actual cell.

$$
\begin{aligned}
\frac{\partial V_{\mathrm{hhl}}}{\partial x} & \approx \frac{\partial V_{r}}{\partial x}-\frac{\partial V_{r}}{\partial z} \cdot h_{h} \\
& =\frac{1}{s}\left(-V_{x-1, y, z}+V_{x+1, y, z}-\frac{-V_{x, y, z+1}+V_{x, y, z-1}}{h_{v}} \cdot h_{h}\right)
\end{aligned}
$$

\subsubsection{Converting from geographical altitude to Index}

Converting between the altitude in the model's atmosphere and the data index that maps the data into the model is a task that is done countless times during the integration of trajectories. In fact, this conversion is responsible for a main part of the algorithms run time since the conversion is not trivial. This is why this conversion needs to be implemented with computational efficiency in mind.

Given the position in the model's atmosphere as $(x, y, z)$ we want to find the vertical index that maps to this data point. As described in Section 4.2.2 the data in the vertical is sampled in irregular distances. An additional challenge is the half-level sampling of the altitude file.

Figure 4.6 visualizes schematically how the index that map's to the position in the model's atmosphere can be found. In the 60 levels provided by the dataset by using a binary search to find the upper bound this results in an average of 5.9 lookups for each request.

Given the coordinates of a horizontal location and the altitude in meter, we need to convert this into the index in the data domain. Because the altitude index is an ordered set we can use a binary search to find the cell index where the point is located and then interpolate the value belonging to the location.

\subsubsection{Interpolation}

To interpolate a value between cells we need to incorporate the different altitude levels of neighboring cells. Instead of using a direct trilinear interpolation we first approximate the altitude level using the algorithm described in Section 4.2.6 of each cell using linear interpolation. Then we use bilinear interpolation in this horizontal plane as visualized in Figure 4.7. 


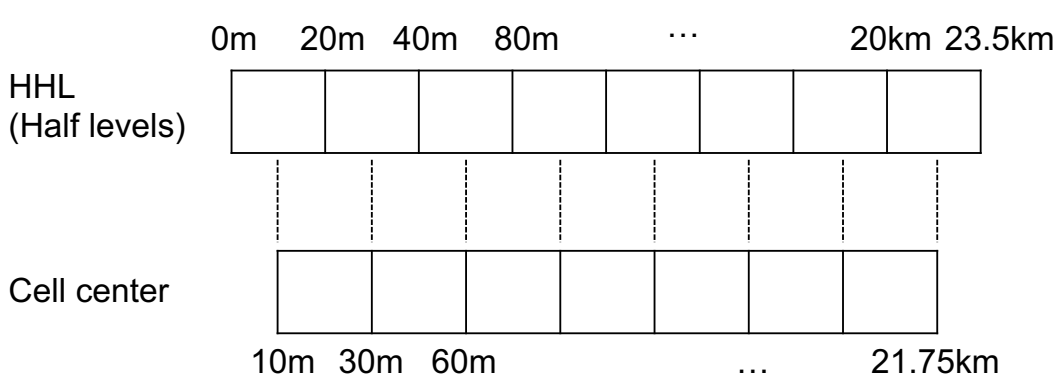

Given altitude $z$ find upper bound, then interpolate

Figure 4.6.: Visualization of the altitude conversion algorithm. Given the half level sampled altitudes we first derive the cell center's altitudes. We want to find the index that maps the data to a point $(x, y, z)$ in the model's atmosphere. The algorithm searches for this point in the ordered altitude set by finding the cell center whose altitude is above the $z$. This enables us to interpolate the index.

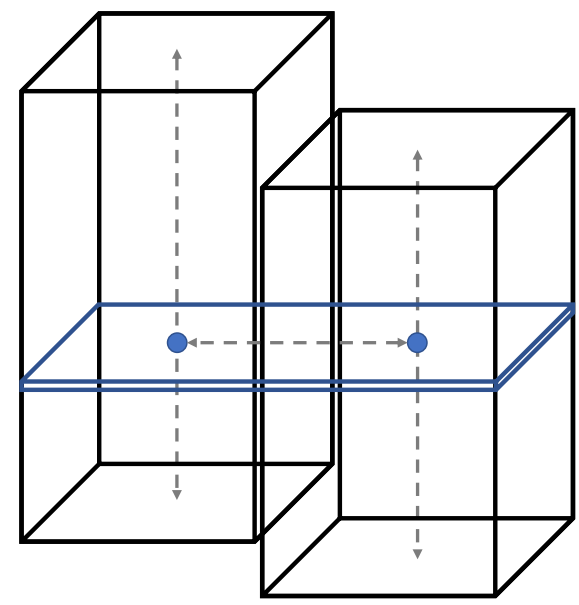

Figure 4.7.: To interpolate the values in the irregular grid we need to first interpolate the vertical components on a horizontal plane and then use bilinear interpolation.

\subsection{Software Architecture}

The PV banner extraction is implemented in $\mathrm{C}++$ and the Visualization Toolkit VTK (Kitware, Inc., Clifton Park, NY). The existing data processing pipeline of $V T K$, as well as the existing visualization functionality for volume rendering and geometry rendering, provided a suitable infrastructure. The graphical user interface was designed using the cross-platform development kit QT and the node based user interface was implemented using QT Node Editor [ea17].

The developed prototype is structured into the three layers presentation, processing and data as visualized in Figure 4.8.

Presentation-layer Interface between the underlying algorithms and the user interface of the application. It consists of the node-based editor as well as the 3D- and 2D-visualization tools. Through a shared scene they are able to process shared events which enables the 


\section{Implementation}

linked views to update each other. Furthermore, the presentation-layer offers a commandline interface to handle preprocessing requests that can be executed in the command line.

Processing-layer Coordinates requests from the presentation layer and executes the required algorithms by forwarding the input data. It moves and processes data between the two surrounding layers while it wraps VTK data structures to enable direct access on the underlying memory structure and it caches requests, either in memory or on disk, to enhance fast responses on repeated requests. This layer is responsible for the handling of the data sampling as described in Sec. 4.1 and computes the trajectory integrations and clustering necessary for the extraction method.

Data-layer Retrieves and stores requested data from the file system and passes it to the processing-layer. This includes the persisted data from the preprocessing as well as intermediate data cached on the disk.

In the following, further details to the software architecture and the three layers is given.

\subsubsection{Presentation-Layer}

The presentation-layer processes the user interactions and serves as interface to the underlying algorithms. It is divided into three parts, namely the node based editor, the rendering of visualizations and the command line interface to access the preprocessing of the application.

\section{Node based interface}

The demand for a flexible and complex composition of different algorithms, filters, and visualization techniques led us to incorporate a data flow paradigm that internally represents the data processing pipeline as a directed graph as described in Section 3.5.4. Figure 4.9 visualizes the execution model of the underlying graph. The execution can be summarized as followed. On request for an update by the user each input node gets notified and starts processing its request by collecting the configuration and forwarding it to the processing-layer. The generated output is forwarded to their direct predecessors. As soon as the notified predecessor has collected all the input data it starts processing the input by requesting its output from the processing-layer. Finally, the generated data is visualized using a sink node. The visualization can incorporate a multitude of different visualization types as listed in Chapter 3.5. All the nodes used throughout this document, including their input and output data type, are listed in Table A.1. Each node can be configured independently by user-defined settings. Because of the deterministic execution of the PV banner extraction algorithm as described in Chapter 3 we can persist the graph and reload its state at a later time. Additionally, the graph can be used as input for the caching functionality of the application to retrieve previously generated output which enables a more interactive experience for the users. 


\section{Rendering using VTK}

Sink nodes are used for the visualization of the generated data and the extracted features. Each sink node requests the visualization object, the so-called actors, from the processing-layer which returns a $V T K$-actor object that can be interactively visualized on the screen. The application supports the visualization of geometry as a line or as isosurfaces that are extracted from scalar volumes. Furthermore, scalar volumes can be rendered using volume renderings (e.g. Figure 5.6) which supports color transfer functions and opacity transfer functions. This variety of different $3 \mathrm{D}$ rendering techniques allows for a multitude of different visualizations. Scalar data can also be extracted as slices and is then visualized as 2D-plots (e.g. 5.6d).

Combining the different rendering possibilities with each other enables a deep insight into the data and their correlations to each other (e.g. Figure 4.10 (a) and (b) shows how we can use a $2 \mathrm{D}$ scatter plot in combination with a 3D volume rendering to extract areas where it is raining and the Potential Vorticity is above a certain threshold). The underlying scene that is based on the directed acyclic graph inherently combines each filter and visualization technique with each other which allows the visualization techniques and filtering possibilities to be combined with each other.

\section{Preprocessing}

Additionally, a command line preprocessing interface enables the direct access to some algorithms which can be used for preprocessing of the data.

\subsubsection{Processing-Layer}

The processing-layer is the core of the developed application. It is responsible for the processing of the user interface and it executes all developed algorithms implemented during this thesis.

Due to the geographic data which is not supported directly by VTK a wrapper for the data was developed which abstracts the data access from the user (as described in Section 4.1). This wrapper allows direct access on the memory to bypass the VTK interface which improves the performance. The wrapper was designed that parallel access to the data is possible and enables caching friendly access to the memory. The algorithms are parallelized using the OpenMPInterface (Open Multi-Processing API).

To enable the caching functionality we take advantage of the determinism of the developed algorithms. This ensures that on a given input the algorithms produce the same output. The directed graph which serves as input for the algorithms can be converted into a hash-value implemented using a custom hash function. Using this hash value the application searches on the disk for previously persisted data based on previously executed algorithms. 


\section{Implementation}

\subsubsection{Data-Layer}

The task of the data-layer is to load data from disk into memory. This requires the layer to process several file formats including the NetCDF file format used for numerical weather simulation data by the IAC and the VTK XML File format used for compressed data access of preprocessed files. Due to the fact that the whole applications loading time is bound to the loading time on disk and not the processing time it is advantageous to store the data in a compressed version to enable faster loading. 


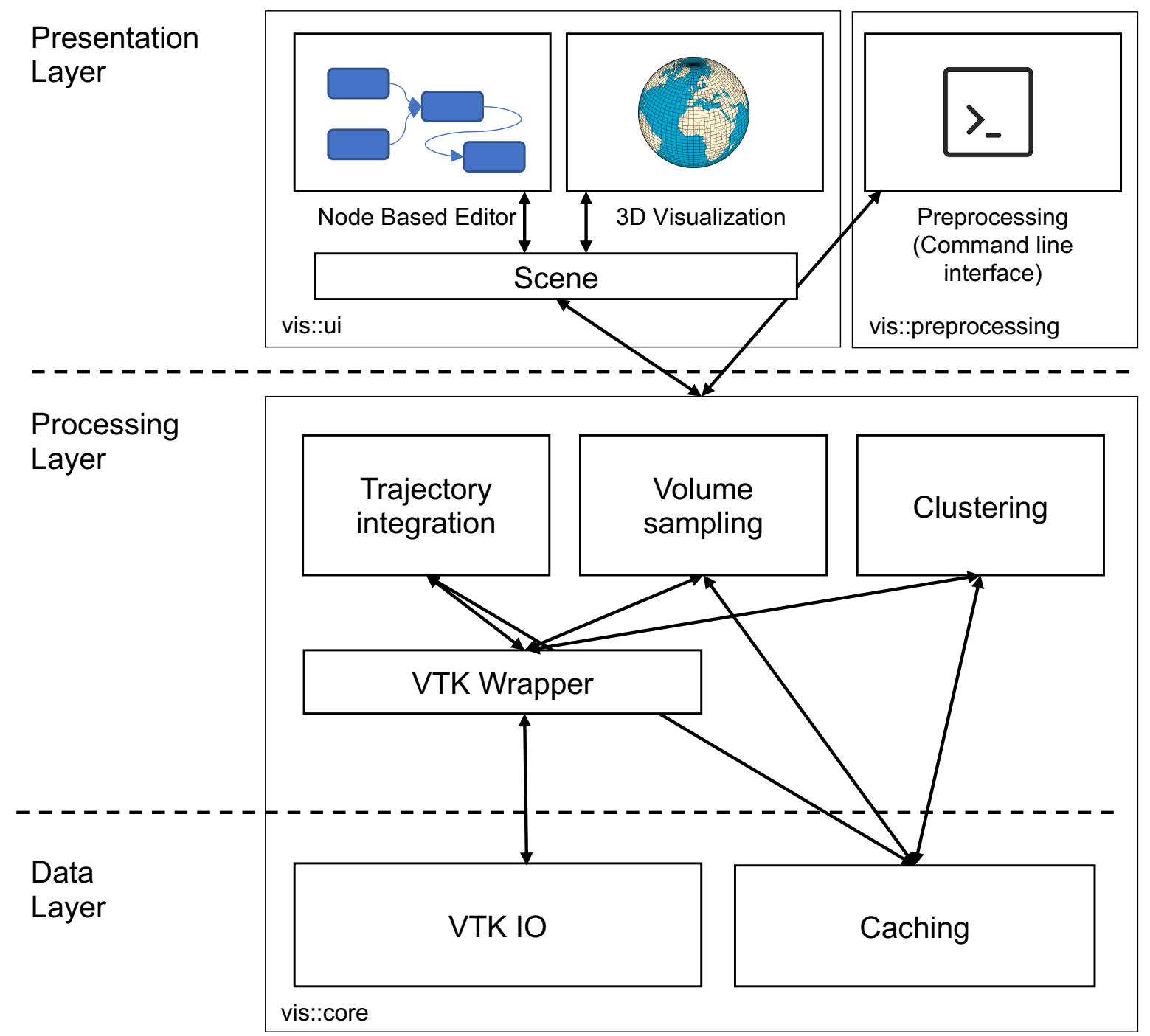

Figure 4.8.: The presentation-, processing- and data- layers build the foundation of the software architecture. 


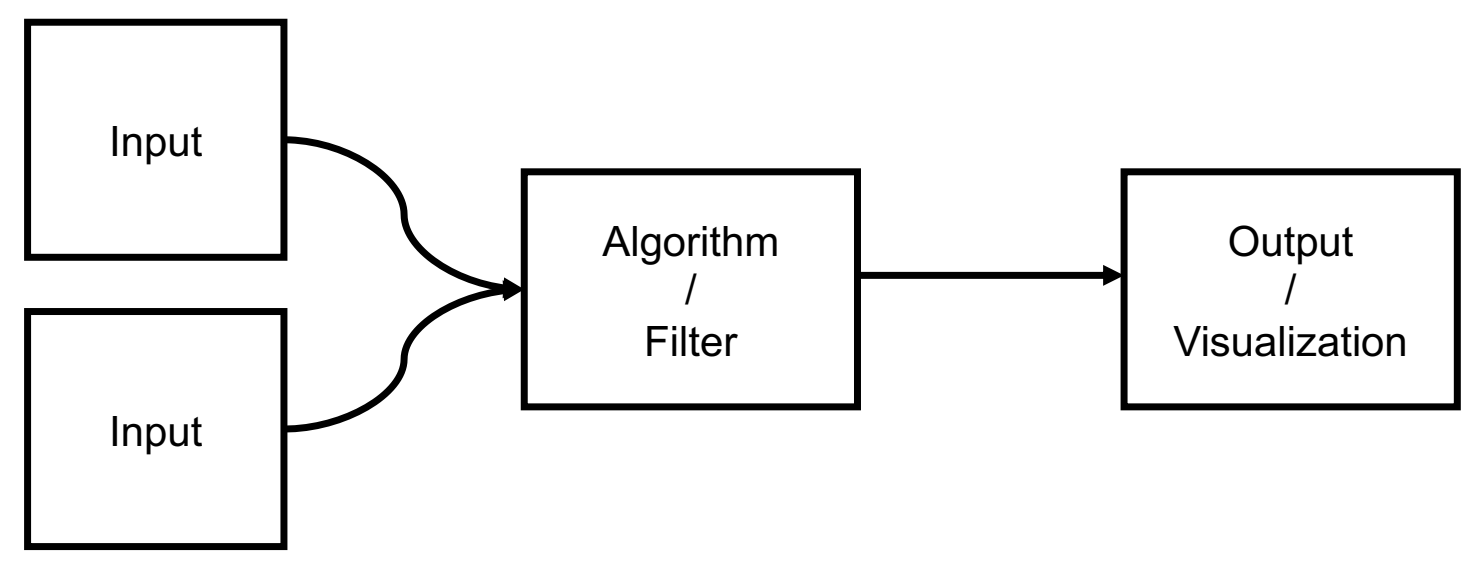

Figure 4.9.: The data flow in the directed graph of the node based editor is processed from input- to output-nodes.

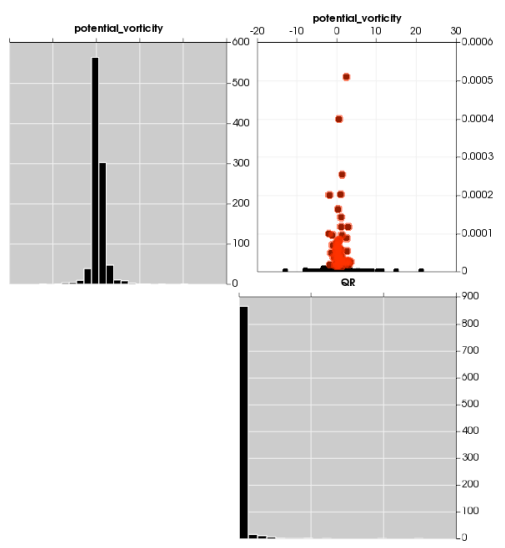

(a)

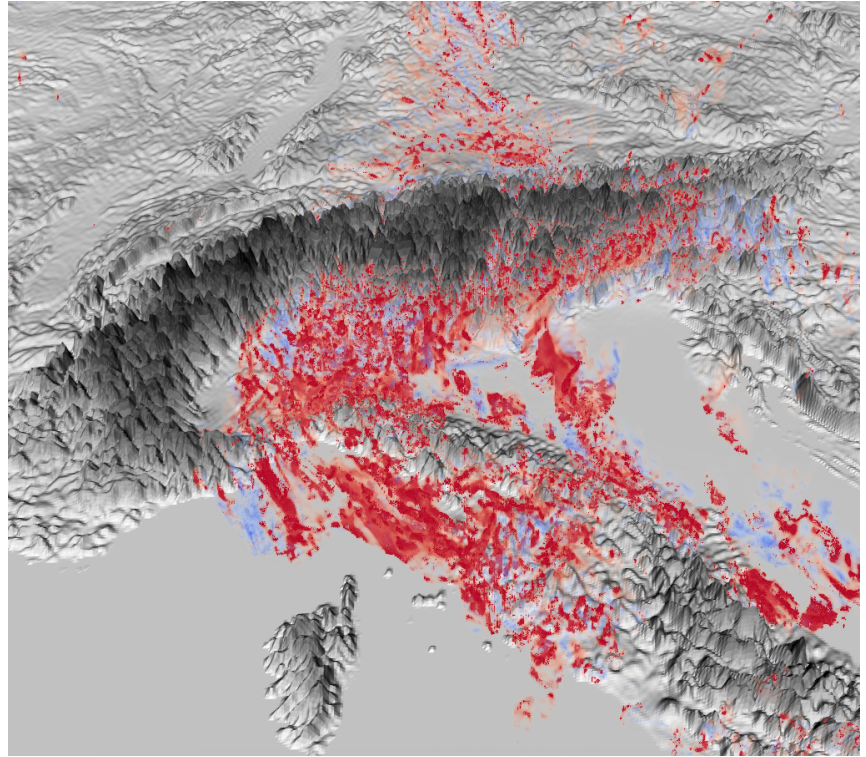

(b)

Figure 4.10.: (a) Using a scatter plot matrix we can select the $P V$ values in cells that also have rain. (b) The selected $P V$ values are visualized using a volume rendering. 


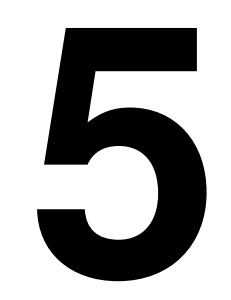

\section{Results}

We demonstrate the extraction method and the developed framework by analyzing two real-world datasets. In the remainder of this section, we provide an overview of the climate simulation and analyze specific aspects of the data. We conclude the presented work by giving an insight into the development process, the design lessons learned, a performance analysis and finally a summary and conclusion.

\subsection{Analysis}

By applying the presented visualization methods we are able to get a deeper understanding of the PV banners and their meteorological surroundings. We present our findings by first analyzing distinct banners, then investigate their structure over the Alps and the downstream topography, and finally, we observe their life cycle in the temporal dimension.

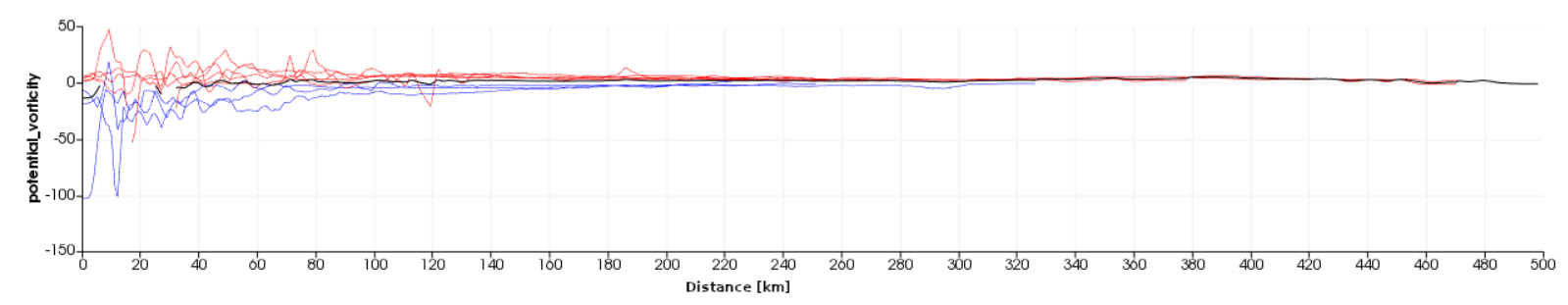

Figure 5.1.: $P V$ value along the extracted core lines. The absolute $P V$ value declines with the length of the banner. Typically the neighboring PV banners with anomalously positive (red) and negative (blue) signs show similar trends along their length. The mean is shown as a black line. 


\section{Results}

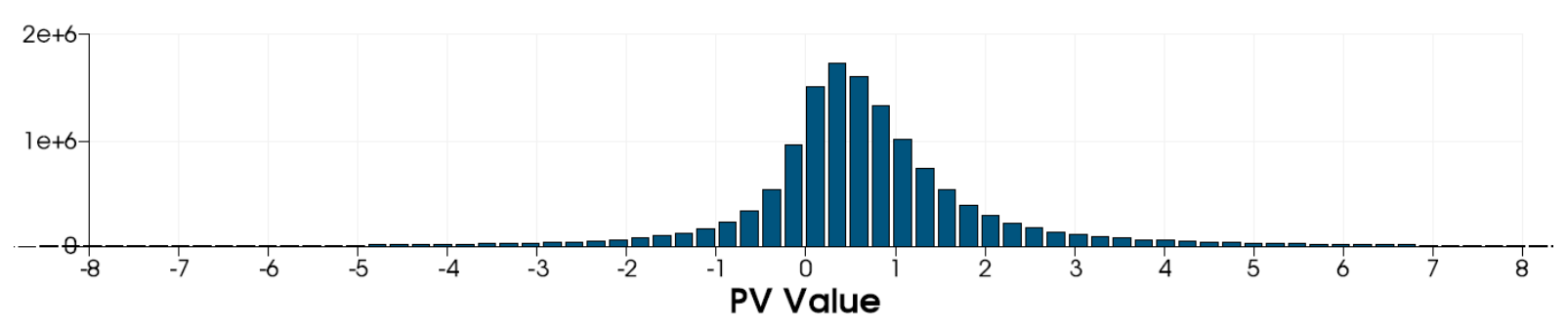

Figure 5.2.: Distribution of $P V$ in the model's atmosphere sampled in cells of constant volume in the altitude range of $0 \mathrm{~km}$ to $6 \mathrm{~km}$ above sea level. The mean of the normally distributed $P V$ values is slightly shifted to the positive side, which was expected by our domain scientists.

\section{Extraction of distinct Potential Vorticity Banners}

The meteorological conditions between the 28 and 29 January 2010 were favorable for the development of PV banners around the Alps due to the strong wind flowing from north to south above the full extent of the Alps. An overview of the 28 January 2010 12:00 UTC is given in Figure 1.1 and Figure 1.2 where the PV banners extend several hundred km downstream.

We extracted a subset of PV banners (see Figure 5.3 (a)), originating from neighboring mountain peaks in the west of the Alps where the topography downstream enables the banners to extend undisturbed. The extracted banners confirm known patterns, namely PV values of anomalously positive and negative sign and PV values declining with the length of the banners. Figure 5.1 illustrates the PV values along the extracted core lines. The neighboring PV banners, which typically differ in their sign, show a similar decay of PV along their length. This results in a linear structure of the mean values of the extracted PV banners. Comparing this with the PV distribution of the model's atmosphere (see Figure 5.2) we observe that the PV values at the source of the banners deviate significantly from the mean.

After the flow detaches from the mountain peaks, the PV banners show a wave-like vertical displacement which Figure 5.3 (c) outlines through the fluctuating altitude changes of the PV banners. It turns out that this pattern can also be observed in the vertical component (Figure 5.3 (b)) of the velocity. The source of these motions is due to atmospheric gravity (buoyancy) waves. These are generated when a stably stratified fluid is forced to flow over a topographic barrier, thereby producing vertical motions. Depending upon the upstream vertical profiles of velocity and density, the wave energy may be trapped and the flow response then exhibits quasi-stationary waves with a well-defined horizontal wavelength. The occurrence of such waves in flow past topography is common, but to our knowledge, their interaction with PV banners has not been noted previously.

The extraction of PV banners facilitates the analysis of their relationship with the atmospheric environment. Adding a horizontal cross section of relative humidity as shown in Figure 5.4 shows a specific example where a correlation of PV banners and other meteorological variables can be identified. The relative humidity is not fully conserved by atmospheric transport, but it nevertheless serves as a tracer of an air mass. The PV banners separate air masses of different origin and thus different relative humidity. Some have flown over the Alps and carry along comparatively high humidity associated with condensation processes to the north of the Alps, others origin from low-levels to the south of the Alps and exhibit comparatively small humidity. 
However, these relationships are complicated as descending air masses will dry due to adiabatic warming.

The ability to map a multitude of meteorological parameters color-mapped onto the extracted banners or on plane cross sections provide the user with insight into the atmospheric processes along the banners that could not be inferred from the conventional 2D approaches.

\section{Structure of PV Banners over the Alps}

The development of PV banners at a specific mountain peak depends on several criteria. One specific prerequisite for the development of elongated lines is that the model's topography downstream is sufficiently smooth. Their extension direction is highly dependent on the wind direction. Because of this a north- or south-bound flow is favorable for the development of PV banners in the European Alps since the PV banners can develop on the whole mountain range.

Interestingly, PV banners can develop in different directions over several altitude levels. Figure 5.5 shows extracted PV banners color-mapped by their altitude. Using a 3D visualization allows an analyst to easily perceive the vertical staggering and different directions of the banners, as well as the associated crossings (between banners at different altitudes) in a straightforward way, compared to conventional 2D visualization, where several layers would need to be compared to each other. The different directions of the banners are due to different wind directions depending upon the altitude. The resulting crossings of banners are consistent with meteorological expectations but have to our knowledge not been shown previously.

Besides PV banners, it is possible to have a PV accumulation in the atmosphere created by processes other than flow detachment around mountains or their peaks. One specific example can be observed in the northwest of Figure 1.2 (b) where the accumulation is caused by a meteorological front. Because of this, it is required that our extraction algorithm can differentiate robustly between PV banners, i.e., PV accumulations that are created from flow detachment, and other PV accumulations.

\section{Time-dependent Analysis}

The life cycle of PV banners starts with their creation and ends with their decay. Typically they have a lifetime of several hours. Responsible for their decay can be a multitude of meteorological processes:

- Wind speed at source drops

- Along their extent the wind downstream disturbs their development

- The wind direction changes over rough topography which does not allow the development of PV banners

When they reach their end of life they typically reduce their length and vanish or the banner decays into parts which are advected downstream by the wind. One such example is shown in Figure 5.6 which shows the decay of a group of PV banners over a time period of 5 hours. This decay was caused by the wind downstream which changed the direction disturb the development 


\section{Results}

of the banners. The banners start to deviate from their course and the banners start deattaches from their source.

The temporal accumulation in Figure 3.6 showed for the time period of 8 hours a temporal accumulation of the PV banners length projected onto the surface. This allows the users to get good temporal overviews of the dataset. In the mountain range on the west, we see the source points of long primary banners which are in the extent of the whole mountain range. Furthermore, several high peaks show increased lengths compared to their surroundings which further emphasizes the effect of flow detachment at the mountain peaks.

\subsection{Development Process \& Design Lessons}

We carefully designed the application in a tight feedback loop with our collaborators. This allowed us to develop the system through repeated cycles (iterative) and in small portions at a time (incremental), allowing the developers to take advantage of what was learned during the development of earlier versions of the tool.

The iterative process involved a requirement analysis, which defined the project goals. In an elaboration process a first prototype was developed which enabled the domain specialists to get first insights of the advantages and additional possibilities provided by $3 \mathrm{D}$ visualizations of climate data. This allowed the team to define the necessary visualization techniques and refine the requirements. The parallel development of the interactive visualizations enabled an agile change process to collectively refine the requirements and adapt the application.

A specific example where this agile reaction on changes was required is in the implementation of the data flow programming model. The close cooperation between the domain scientists and developers revealed that the prototyped application did not enable the degree of freedom to combine different algorithms and visualization techniques, as desired. The user interfaces were too complicated to allow complex and flexible filtering. This led us to research other possibilities to provide the end user with better access to the underlying algorithms and data. The final decision to satisfy this requirement was to implement the node-based data flow programming model.

\subsection{Performance}

This section reports on the performance of our approach. The focus is on the computation time since the memory consumption to store PV banners is negligible compared to the simulation data size. Our method depends on the precalculation of the potential vorticity, a scalar value which is not necessarily available in a meteorological dataset. The computation time to extract banners is inherently dependent on the number of seed points which determines the number of lines to be integrated. The PV line computation is parallelized since the extraction of each banner candidate is independent. All timings are taken on a workstation with an Intel Core i7 8700 processor with $3.2 \mathrm{GHz} \times 6$ cores, $16 \mathrm{~GB}$ RAM and a NVIDIA GeForce GTX 1050TI.

The most time consuming part is the gradient calculation and the gradient descent method used 


\begin{tabular}{llll}
\hline \# Seedpoints & $2000^{\prime} 000$ & $500^{\prime} 000$ & $10^{\prime} 000$ \\
\hline \#Banner & $13^{\prime} 764$ & $3^{\prime} 430$ & 519 \\
\# Cluster & 996 & 617 & 252 \\
Integration time & $9^{\prime} 300 \mathrm{~ms}$ & $2^{\prime} 300 \mathrm{~ms}$ & $352 \mathrm{~ms}$ \\
Cluster time & $880 \mathrm{~ms}$ & $100 \mathrm{~ms}$ & $10 \mathrm{~ms}$ \\
Visualization time & $10 \mathrm{~ms}$ & $3 \mathrm{~ms}$ & $1 \mathrm{~ms}$ \\
Precomputation of PV & $3700 \mathrm{~ms}$ & & \\
\hline
\end{tabular}

Table 5.1.: The timings were measured on a grid with $500 \times 500 \times 60$ resolution, based on the node graph earlier shown in Figure 3.8.

to correct the banners. This is particularly expensive due to the conversion of the surface aligned model levels to meters. The method enables high scalability to larger datasets. Limits could be pushed further by employing a GPU integration or by distributing the work on several machines. 


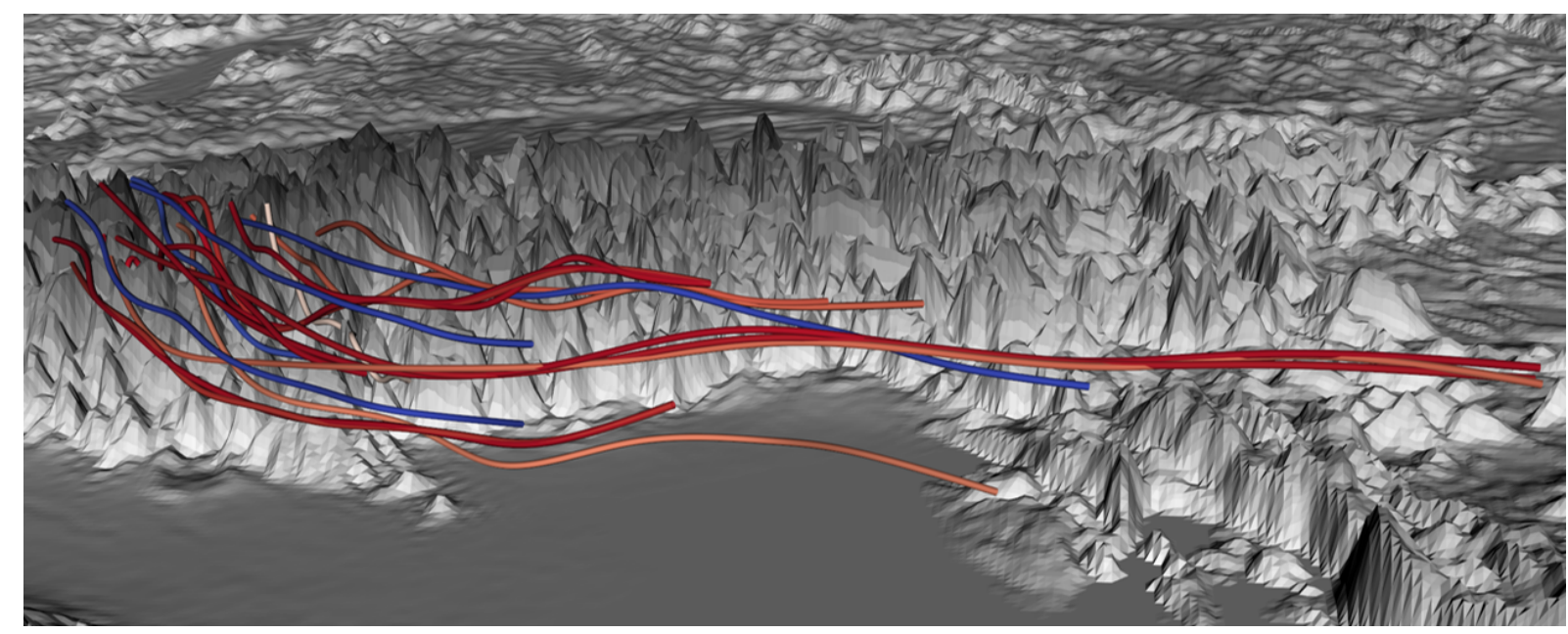

(a)

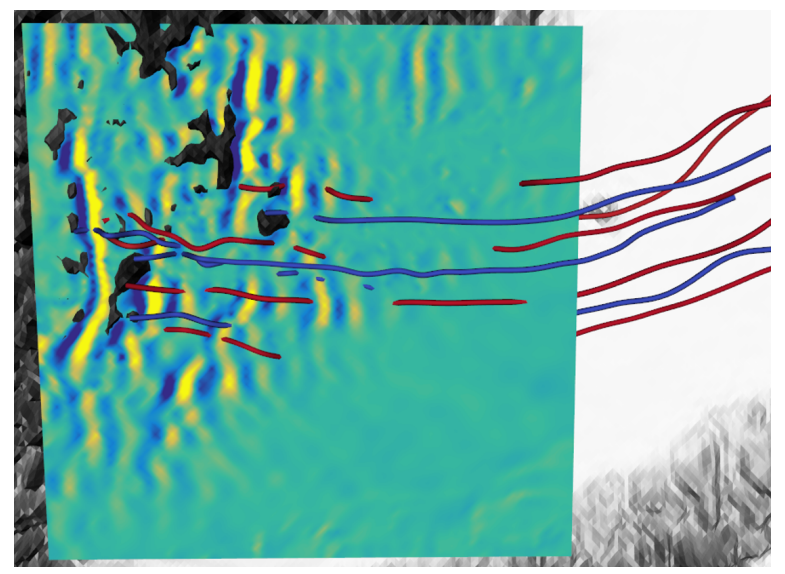

(b)

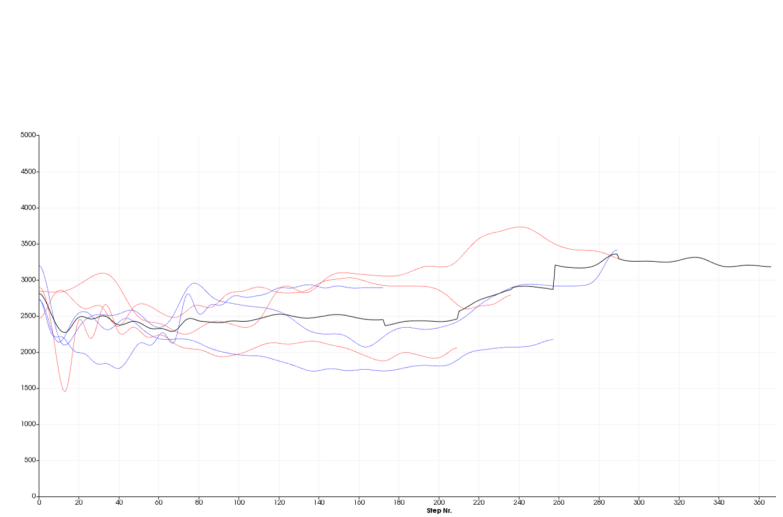

(c)

Figure 5.3.: (a) Selected $P V$ banners extracted to analyze their spatial extent. (b) Horizontal cross section of the vertical wind component at fixed altitude along the $P V$ banner. The wave-like patterns in the flow direction (from left to right) is associated with atmospheric gravity (or buoyancy) waves and is responsible for the altitude changes of $P V$ banners. (c) Altitude of the $P V$ banners along their full extent. The banners show an undulating pattern in the altitude change close to their source. Color-coded positive (red) and negative (blue) banners. 


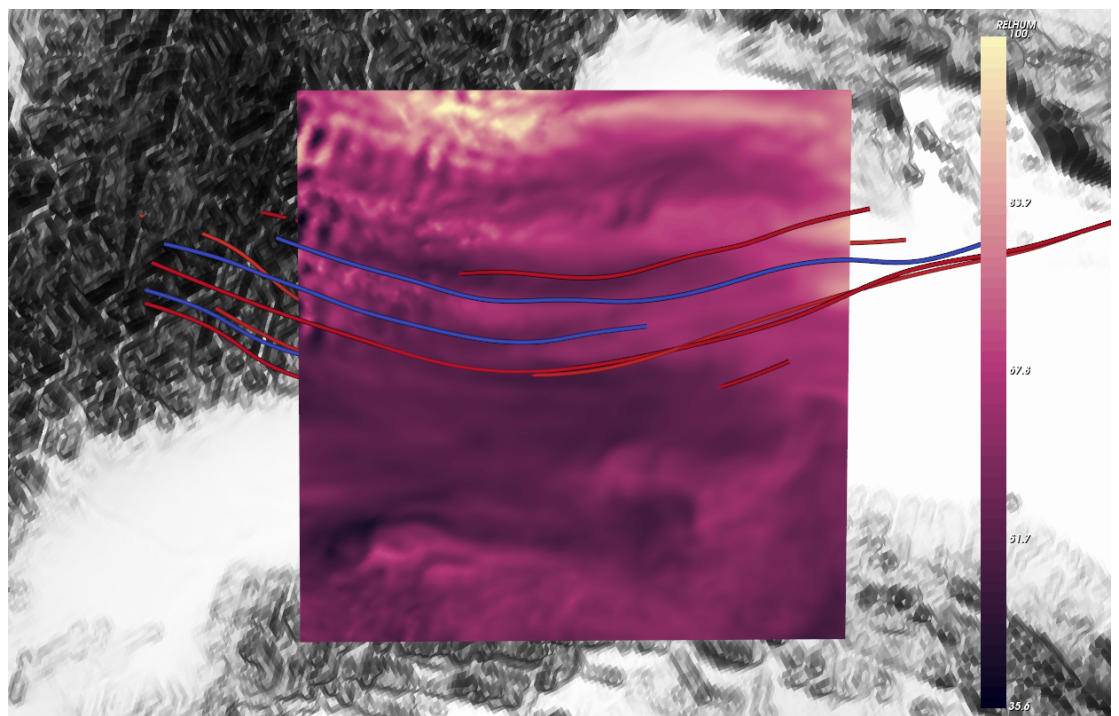

Figure 5.4.: Relative humidity extracted as a horizontal cross section around the $P V$ banners. A correlation between the $P V$ banners and relative humidity values is visible. This highlights that $P V$ banners separate air masses of alternating high and low humidity.

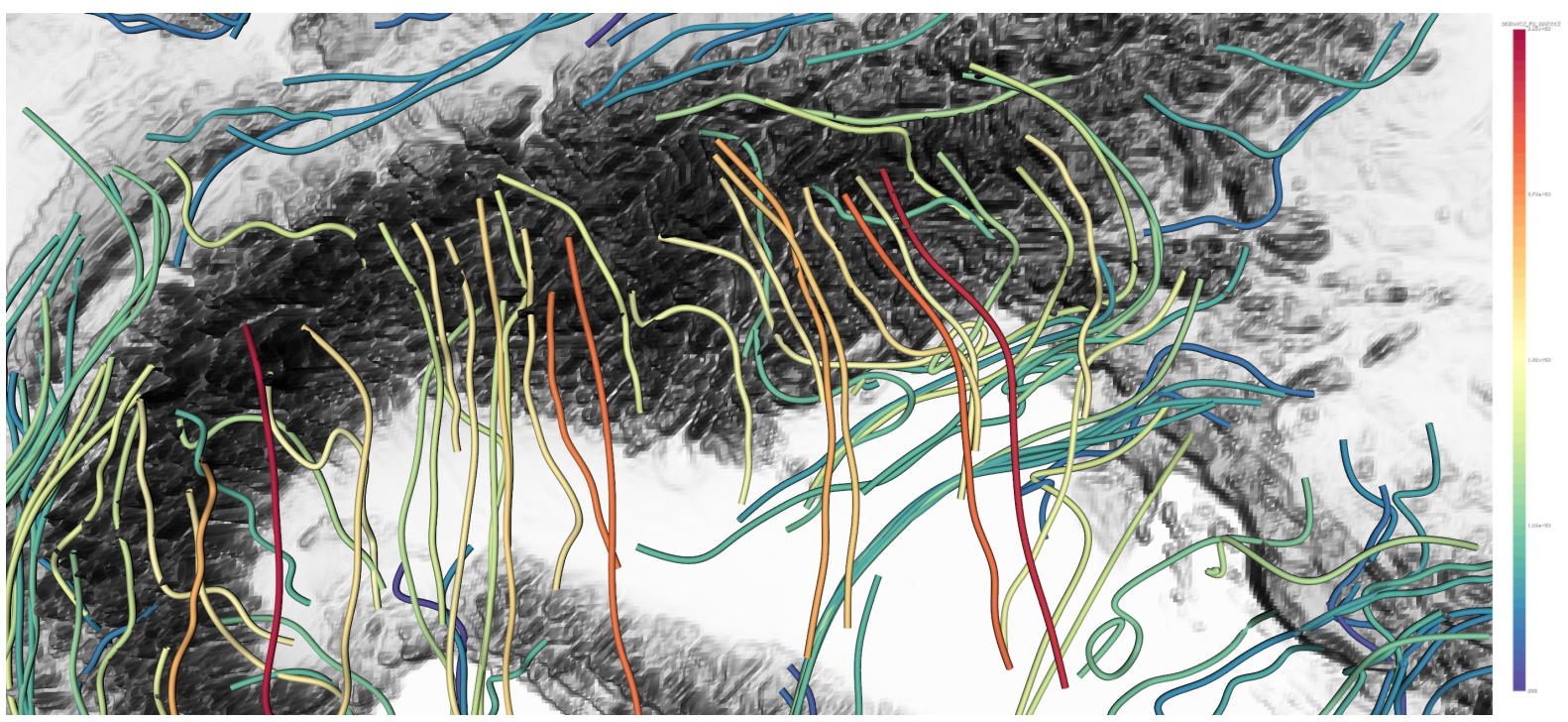

Figure 5.5.: $P V$ banners color-mapped by altitude. Banners at different altitudes exhibit different directions and may cross each other. Comprehending these crossings is straightforward in our $3 D$ visualization, but difficult to detect in standard horizontal displays. 27 January 2010 20:10 UTC. 


\section{Results}

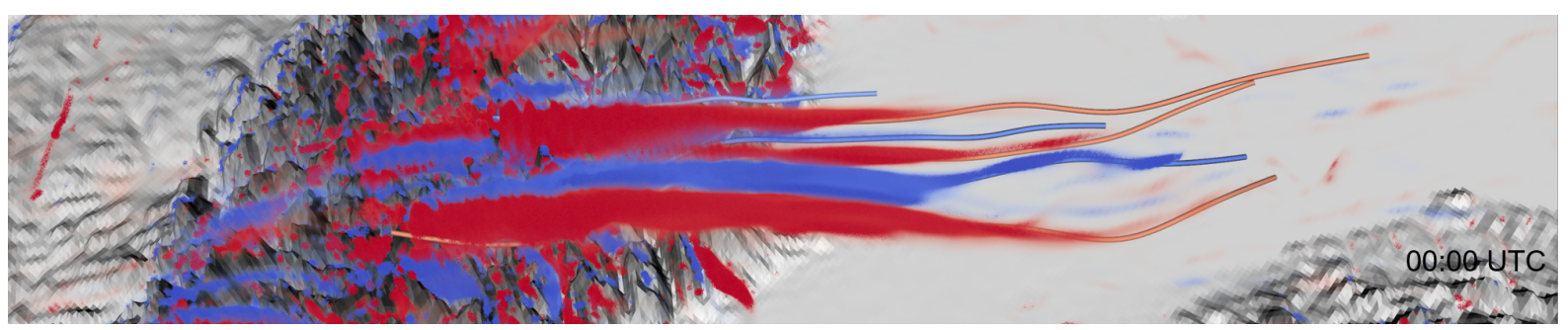

(a)

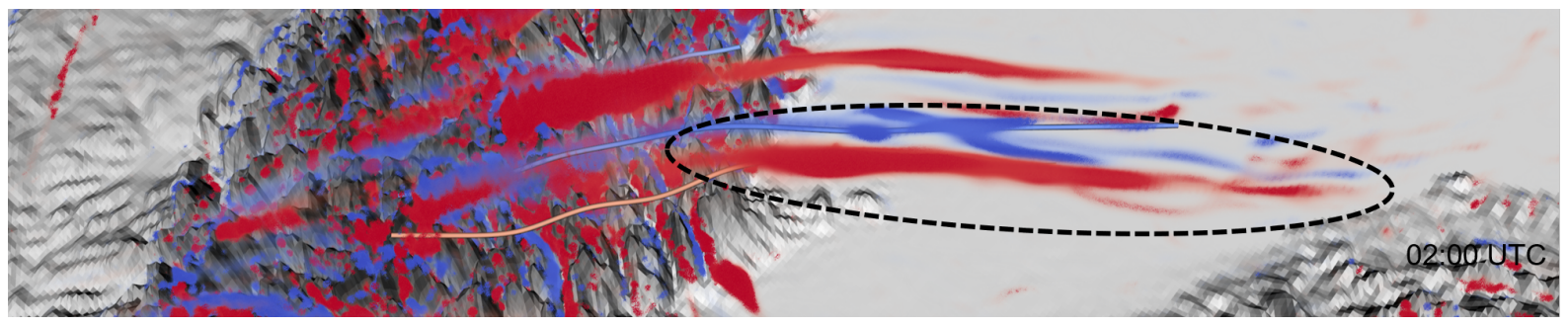

(b)

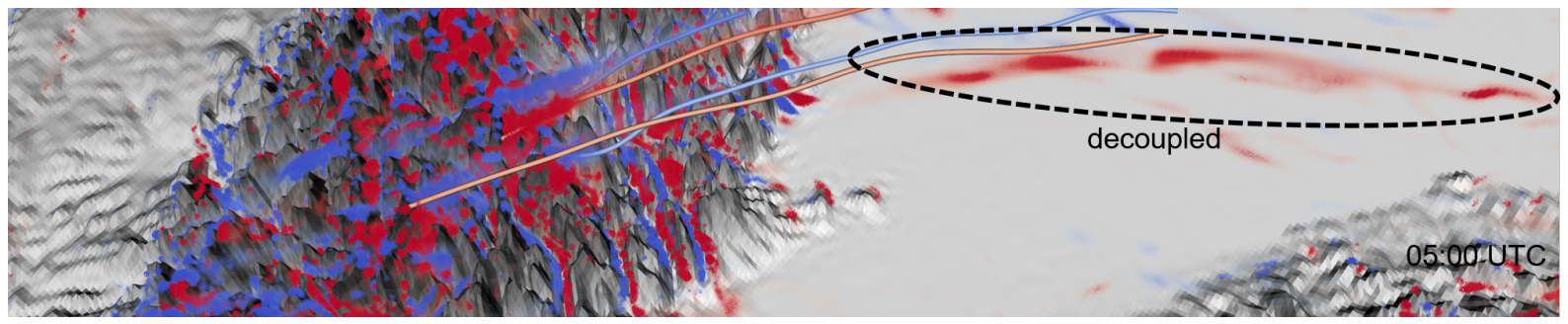

(c)

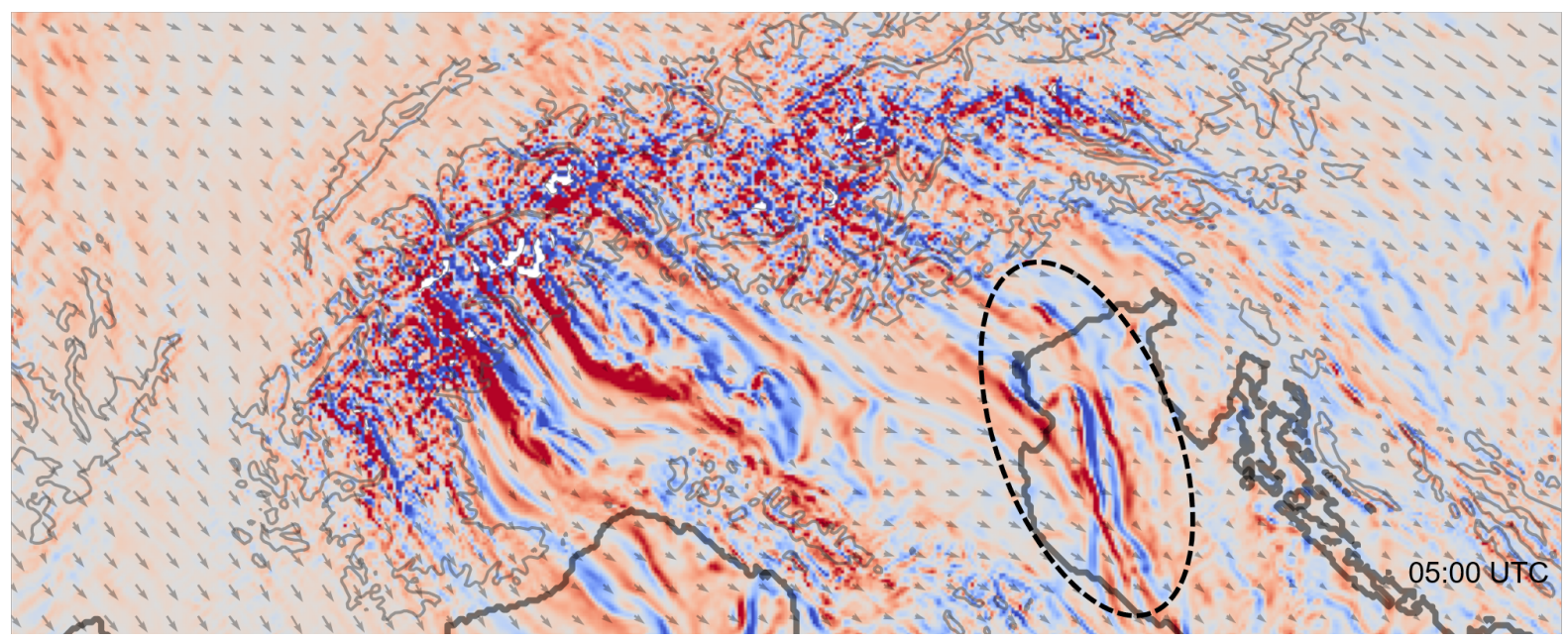

(d)

Figure 5.6.: 29 January 2010 from 00:00 to 05:00 UTC. End of lifetime sequence of PV banners which are rendered as volumes with the corresponding core lines as geometrical lines. (a) The wind flows from north (left) to south (right). The wind direction in the Po valley changes eastwards which is responsible for the eastward bend in their end. (b) The PV banner start to decouple from the mountains source. (c) The decoupled PV banners are advected with the wind flowing over the Alps. (d) Horizontal cross section of the PV banners on $3000 \mathrm{~m}$ above sea level which emphasizes the decoupling of the PV banners. 


\section{6}

\section{Conclusion and Future Work}

We presented a method to extract potential vorticity banners from numerical weather prediction data. The method is based on the integration based predictor-corrector [BS94] algorithm to find extremal lines in the PV data. Our method is able to robustly extract the extremal lines over long distances and through noisy data. We place the seed points above the terrain to filter out PV accumulations that are not based on orographic flow splitting. Using Agglomerative Hierarchical Clustering we are able to combine different banners that converge into the same banner structure. We implemented a workflow that enables the user to interactively identify PV banners and to track them through time. To facilitate the analysis we provide several visualization techniques such as line visualizations and information visualizations supporting linking and brushing. We demonstrated the framework on the analysis of PV banners in two numerical datasets, highlighting the value of our method.

In close collaboration with the domain scientists, we designed an application that fulfills the given requirements to facilitate further research on PV banners. The time needed to compute the banners is within seconds to a few minutes and the analysis of the data can be done at interactive frame rates. Our approach makes the data more accessible since it becomes easier to interpret the spatial structure of the extracted line features, compared to the currently used 2D visualizations. The method is able to remove visual clutter and enables the observation of PV banners over time. This is of crucial importance to understand the spatial structure of PV banners, to explore the relationship between the banners and their environment and to study the life cycle of PV banners. In particular, we shed light onto two inherently three-dimensional processes that have previously not been visualized: the vertical crossing of potential vorticity banners in different altitude levels, and their vertical waviness in the lee of the mountains.

A limitation of our approach is that it produces an overabundance of seed points which have to be clustered to representative banners. The overabundance of seed points results in additional computational effort and additional filtering is necessary in a post-processing step.

The meteorologists hypothesized that there is a strong correlation between PV banners and 


\section{Conclusion and Future Work}

precipitation as previous meteorological research suggested a link between the terrain introduced PV and banded convection [SG16] as convective cloud bands were aligned parallel to these PV banners. The two case studies that were examined throughout this Thesis did not show a strong correlation between PV banners and precipitation.

Future work might include the extraction of tubes around core lines which would allow further insight into the surroundings of PV banners. This is a challenging task as the tubes are not always spherical and they can show strongly varying shapes along the vertical axis. Furthermore, PV banners differ from each other by their PV values which does not allow to search for a constant value which defines if a point is within a banners tube. This could be solved, for example, by observing the gradient from the cell center to the tubes boundaries and terminating the tube at a point of high decrease of the PV value.

A further interesting extension to this project would be to conduct a user analysis on how the 3D visualizations and user interface are perceived within the meteorological community. Previous research on the adoption of 3D visualization applications showed [SGMM03] that meteorologists are reluctant to adapt to new visualization methods as they have long ingrained traditions. The feedback from the meteorologists at IAC was very positive. A well received feature are the interactively placeable cross-sections and the interactivity of the developed 3D applications. 


\section{A}

\section{Appendix}

\section{A.1. Filter, algorithms and node types of application}

Following table lists the implemented nodes used throughout this paper.

\begin{tabular}{|c|c|c|c|}
\hline Node Name & Functionality & Input & Output \\
\hline Animation & Requests sequential time steps from input & Animation & - \\
\hline Banner End Filter & $\begin{array}{l}\text { Early termination of extracted PV banners based } \\
\text { on PV value }\end{array}$ & Trajectory & Trajectory \\
\hline Box Widget & $\begin{array}{l}\text { Generates implicit function of box placed in } \\
\text { scene }\end{array}$ & - & $\begin{array}{l}\text { Implicit } \\
\text { Shape }\end{array}$ \\
\hline Cluster Analyzer & Visualization of cluster data & Trajectory & - \\
\hline $\begin{array}{l}\text { Cluster Min. Size } \\
\text { Filter }\end{array}$ & Filters clusters that have not enough members & Cluster & $\begin{array}{l}\text { Trajectory, } \\
\text { Cluster }\end{array}$ \\
\hline Cluster to Actor & $\begin{array}{l}\text { Converts cluster data into a 3D visualization ob- } \\
\text { ject }\end{array}$ & Cluster & - \\
\hline $\begin{array}{l}\text { Clustered Trajecto- } \\
\text { ries }\end{array}$ & $\begin{array}{l}\text { (Convenience) Combines predictor corrector \& } \\
\text { clustered trajectories }\end{array}$ & Seed Point & $\begin{array}{l}\text { Trajectory, } \\
\text { Cluster }\end{array}$ \\
\hline Color Bar & Visualization of color bar & Color & - \\
\hline Color Node & Generates color map for visualization & Color Range & Color \\
\hline Delta Time & Requests a time step with a delta time from input & Seed Point & Seed Point \\
\hline
\end{tabular}




\begin{tabular}{|c|c|c|c|}
\hline Node Name & Functionality & Input & Output \\
\hline Extract Selection & Filters input using the mouse selection & $\begin{array}{l}\text { Trajectory, Se- } \\
\text { lection }\end{array}$ & Trajectory \\
\hline Extract Surface & Extracts data from volume along surface & - & Point Data \\
\hline $\begin{array}{l}\text { Filter Seed Points } \\
\text { by Shape }\end{array}$ & Filters seed points by an implicit function & $\begin{array}{l}\text { Seed Point, } \\
\text { Implicit } \\
\text { Shape }\end{array}$ & Seed Point \\
\hline $\begin{array}{l}\text { Filter Trajectory by } \\
\text { Shape }\end{array}$ & Filters trajectories by an implicit function & $\begin{array}{l}\text { Trajectory, Im- } \\
\text { plicit Shape }\end{array}$ & Trajectory \\
\hline $\begin{array}{l}\text { Filter Trajectory } \\
\text { Positive/Negative }\end{array}$ & $\begin{array}{l}\text { Outputs positive \& negative trajectories sepa- } \\
\text { rated }\end{array}$ & Trajectory & Trajectory \\
\hline Path Line & Integrates path lines & Seed Point & Trajectory \\
\hline Point Data to Color & $\begin{array}{l}\text { Computes data range of point data to generate } \\
\text { color maps }\end{array}$ & Point Data & Color Range \\
\hline Point Widget & $\begin{array}{l}\text { Generates a single seed point defined by a point } \\
\text { in } 3 \mathrm{D} \text { scene }\end{array}$ & - & Seed Point \\
\hline $\begin{array}{l}\text { Predictor Correc- } \\
\text { tor }\end{array}$ & $\begin{array}{l}\text { Computes PV banners using predictor / correc- } \\
\text { tor }\end{array}$ & Seed Point & Trajectory \\
\hline $\begin{array}{l}\text { Resample Trajec- } \\
\text { tory }\end{array}$ & $\begin{array}{l}\text { Algorithm that resamples a trajectory to fixed } \\
\text { distances or to fewer/more line elements }\end{array}$ & Trajectory & Trajectory \\
\hline Sample Trajectory & Samples scalar values on trajectories & Trajectory & Trajectory \\
\hline $\begin{array}{l}\text { Seed Point from } \\
\text { Shape }\end{array}$ & Converts an implicit function to seed points & $\begin{array}{l}\text { Implicit } \\
\text { Shape }\end{array}$ & Seed Point \\
\hline Sphere Widget & $\begin{array}{l}\text { Generates implicit function of sphere placed in } \\
\text { scene }\end{array}$ & - & Seed Point \\
\hline Streak Line & Integrates streak lines & Seed Point & Trajectory \\
\hline Stream Line & Integrates stream lines & Seed Point & Trajectory \\
\hline Surface Seed & Creates seed points along surface & - & Seed Point \\
\hline Surface to Glyphs & Visualizes point data in scene & $\begin{array}{l}\text { Point Data, } \\
\text { Color }\end{array}$ & - \\
\hline $\begin{array}{l}\text { Temporal Accumu- } \\
\text { lator }\end{array}$ & $\begin{array}{l}\text { Accumulates data of trajectories over time to cre- } \\
\text { ate temporal summaries }\end{array}$ & Trajectory & $\begin{array}{l}\text { Temporal Tra- } \\
\text { jectory }\end{array}$ \\
\hline Temporal Analysis & Visualization of temporal summaries & $\begin{array}{l}\text { Temporal Tra- } \\
\text { jectory }\end{array}$ & - \\
\hline
\end{tabular}




\begin{tabular}{|c|c|c|c|}
\hline Node Name & Functionality & Input & Output \\
\hline Temporal Cluster & Creates temporal clusters of PV banners & $\begin{array}{l}\text { Temporal Tra- } \\
\text { jectory }\end{array}$ & $\begin{array}{l}\text { Temporal } \\
\text { Cluster }\end{array}$ \\
\hline $\begin{array}{l}\text { Temporal Cluster } \\
\text { Actor }\end{array}$ & Visualizes temporal clusters in Scene & $\begin{array}{l}\text { Temporal } \\
\text { Cluster }\end{array}$ & - \\
\hline $\begin{array}{l}\text { Temporal Sum- } \\
\text { mary }\end{array}$ & Visualization of temporal summaries & $\begin{array}{l}\text { Temporal Tra- } \\
\text { jectory }\end{array}$ & - \\
\hline $\begin{array}{l}\text { Temporal Trajec- } \\
\text { tory to Trajectory }\end{array}$ & $\begin{array}{l}\text { Converts temporal trajectories to normal trajec- } \\
\text { tories }\end{array}$ & $\begin{array}{l}\text { Temporal Tra- } \\
\text { jectory }\end{array}$ & Trajectory \\
\hline Trajectory Cluster & Clusters trajectories & Trajectory & $\begin{array}{l}\text { Cluster, } \\
\text { Trajectory }\end{array}$ \\
\hline Trajectory Details & Visualization of trajectory details like length etc. & Trajectory & Selection \\
\hline Trajectory Filter & $\begin{array}{l}\text { Filtering of Trajectories according to user de- } \\
\text { fined rules }\end{array}$ & Trajectory & Trajectory \\
\hline $\begin{array}{l}\text { Trajectory Plane } \\
\text { Extractor }\end{array}$ & $\begin{array}{l}\text { Shows an implicit plane along trajectory in } \\
\text { scene }\end{array}$ & Trajectory & Point Data \\
\hline $\begin{array}{l}\text { Trajectory Sum- } \\
\text { mary Node }\end{array}$ & $\begin{array}{l}\text { Creates summaries of a large amount of trajec- } \\
\text { tories }\end{array}$ & Trajectory & Selection \\
\hline Trajectory to Actor & Visualizes trajectories in Scene & $\begin{array}{l}\text { Trajectory, } \\
\text { Color }\end{array}$ & $\begin{array}{l}\text { Selection, An- } \\
\text { imation }\end{array}$ \\
\hline Trajectory to Color & Extracts data ranges to create a color map & Trajectory & Color Range \\
\hline $\begin{array}{l}\text { Trajectory to Seed } \\
\text { Points }\end{array}$ & Creates seed points along the trajectory & Trajectory & Seed Point \\
\hline $\begin{array}{l}\text { Trajectory to Sur- } \\
\text { face Actor }\end{array}$ & $\begin{array}{l}\text { Visualization of surface projection of trajecto- } \\
\text { ries }\end{array}$ & $\begin{array}{l}\text { Trajectory, } \\
\text { Color }\end{array}$ & Trajectory \\
\hline
\end{tabular}

Table A.1.: List of the implemented node types in application. Input \& output describe the datatype of the nodes. 



\section{Bibliography}

[AS98] Urs Aebischer and Christoph Schär. Low-Level Potential Vorticity and Cyclogenesis to the Lee of the Alps. Journal of the Atmospheric Sciences, 55(2):186-207, 1998.

$\left[\mathrm{BBB}^{+} 01\right]$ P. Bougeault, P. Binder, A. Buzzi, R. Dirks, R. Houze, J. Kuettner, R. B. Smith, R. Steinacker, and H. Volkert. The MAP special observing period. Bulletin of the American Meteorological Society, 82(3):433-462, 2001.

[BH79] D. A. Bennetts and B. J. Hoskins. Conditional symmetric instability âĂ̌̌ a possible explanation for frontal rainbands. Quarterly Journal of the Royal Meteorological Society, 105(446):945-962, 1979.

[BS94] D C Banks and B A Singer. Vortex tubes in turbulent flows: identification, representation, reconstruction. IEEE Conference on Visualization, pages 132-139, CP14, 1994.

$\left[\mathrm{BSB}^{+}\right.$02] R. Benoit, C. Schär, P. Binder, S. Chamberland, H.C. Davies, M. Desgagné, C. Girard, C. Keil, N. Kouwen, D. Lüthi, D. Maric, E. Müller, P. Pellerin, J. Schmidli, F. Schubiger, C. Schwierz, M. Sprenger, A. Walser, S. Willemse, W. Yu, and E. Zala. The Real-Time Ultrafinescale Forecast Support during the Special Observing Period of the MAP. Bulletin of the American Meteorological Society, 83(1):85-109, 2002.

[BSS15] Nikolina Ban, Juerg Schmidli, and Christoph Schär. Heavy precipitation in a changing climate: Does shortâĂ ̌̌term summer precipitation increase faster? Geophysical Research Letters, 42(4):1165-1172, 2015.

[BW11] Maxi Boettcher and Heini Wernli. Life Cycle Study of a Diabatic Rossby Wave as a Precursor to Rapid Cyclogenesis in the North AtlanticâǍŤDynamics and Forecast Performance. Monthly Weather Review, 139(6):1861-1878, 2011. 
[CGG05] I. Corouge, S. Gouttard, and G. Gerig. Towards a shape model of white matter fiber bundles using diffusion tensor MRI. 2004 2nd IEEE International Symposium on Biomedical Imaging: Nano to Macro (IEEE Cat No. 04EX821), pages 344-347, 2005.

[CL93] Brian Cabral and Leith Casey Leedom. Imaging vector fields using line integral convolution. Technical report, Lawrence Livermore National Lab., CA (United States), 1993.

[DUS $\left.^{+} 11\right]$ D. P. Dee, S. M. Uppala, A. J. Simmons, P. Berrisford, P. Poli, S. Kobayashi, U. Andrae, M. A. Balmaseda, G. Balsamo, P. Bauer, P. Bechtold, A. C. M. Beljaars, L. van de Berg, J. Bidlot, N. Bormann, C. Delsol, R. Dragani, M. Fuentes, A. J. Geer, L. Haimberger, S. B. Healy, H. Hersbach, E. V. Hólm, L. Isaksen, P. Kallberg, M. Köhler, M. Matricardi, A. P. McNally, B. M. Monge-Sanz, J.J. Morcrette, B. K. Park, C. Peubey, P. de Rosnay, C. Tavolato, J.N. Thépaut, and F. Vitart. The ERAInterim reanalysis: configuration and performance of the data assimilation system. Quar. J. Roy. Meteor. Soc, 137(656):553-597, 2011.

[ea17] Dmitry Pinaev et al. Qt5 node editor. https://github.com/paceholder/ nodeeditor, 2017.

[Ebe12] David Eberly. Ridges in image and data analysis, volume 7. Springer Science \& Business Media, 2012.

[Ert42] H. Ertel. Ein neuer hydrodynamischer Erhaltungssatz. Naturwissenschaften, (3):543-544, 1942.

$\left[\mathrm{FOL}^{+}{ }^{14}\right]$ Oliver Fuhrer, Carlos Osuna, Xavier Lapillonne, Tobias Gysi, Mauro Bianco, Andrea Arteaga, and Thomas C Schulthess. Towards a performance portable, architecture agnostic implementation strategy for weather and climate models. Supercomputing Frontiers and Innovations, 1(1):44-61, 2014.

[GST16] Tobias Günther, Maik Schulze, and Holger Theisel. Rotation Invariant Vortices for Flow Visualization. IEEE Transactions on Visualization and Computer Graphics, 22(1):817-826, 2016.

[GT18] Tobias Günther and Holger Theisel. The State of the Art in Vortex Extraction. Computer Graphics Forum, 37(6):149-173, 2018.

[HMR85] B. J. Hoskins, M. E. McIntyre, and A. W. Robertson. On the use and significance of isentropic potential vorticity maps. Quarterly Journal of the Royal Meteorological Society, 111(6):877-946, 1985.

[Hos74] B. J. Hoskins. The role of potential vorticity in symmetric stability and instability. Quarterly Journal of the Royal Meteorological Society, 69(300):155-162, 1974.

[HTWS07] Hans-Christian Hege, Holger Theisel, Tino Weinkauf, and Jan Sahner. Cores of Swirling Particle Motion in Unsteady Flows. IEEE Transactions on Visualization and Computer Graphics, 13(6):1759-1766, 2007.

[JHM04] Wesley M. Johnston, J. R. Paul Hanna, and Richard J. Millar. Advances in dataflow programming languages. ACM Computing Surveys, 36(1):1-34, 2004. 
[ $\left.\mathrm{KCH}^{+} 18\right]$ G Kindlmann, Charisee Chiw, Tri Huynh, Attila Gyulassy, John Reppy, and PT Bremer. Rendering and extracting extremal features in 3D fields. Computer Graphics Forum (Proc. EuroVis), 37(3):525-536, 2018.

$\left[\mathrm{KHS}^{+}{ }^{18}\right.$ Michael Kern, Tim Hewson, Filip Sadlo, Rudiger Westermann, and Marc Rautenhaus. Robust Detection and Visualization of Jet-Stream Core Lines in Atmospheric Flow. IEEE Transactions on Visualization and Computer Graphics, 24(1):893-902, 2018.

[KSS01] N. Kljun, M. Sprenger, and C. Schär. Frontal modification and lee cyclogenesis in the alps: A case study using the alpex reanalysis data set. Meteorology and Atmospheric Physics, 78(1-2):89-105, 2001.

[LFL $\left.{ }^{+} 16\right]$ David Leutwyler, Oliver Fuhrer, Xavier Lapillonne, Daniel Lüthi, and Christoph Schär. Towards European-scale convection-resolving climate simulations with GPUs: A study with COSMO 4.19. Geoscientific Model Development, 9(9):33933412, 2016.

[LLB ${ }^{+}$17] David Leutwyler, Daniel Lüthi, Nikolina Ban, Oliver Fuhrer, and Christoph Schär. Evaluation of the convection-resolving climate modeling approach on continental scales. Journal of Geophysical Research, 122(10):5237-5258, 2017.

[MK97] Hideaki Miura and Shigeo Kida. Identification of Tubular Vortices in Turbulence. Journal of the Physical Society of Japan, 66(5):1331-1334, may 1997.

[MPW13] A. K. Miltenberger, S. Pfahl, and H. Wernli. An online trajectory module (version 1.0) for the nonhydrostatic numerical weather prediction model COSMO. Geoscientific Model Development, 6(6):1989-2004, 2013.

[NSW99] Thomas Nocke, Till Sterzel, and Markus Wrobel. Visualization of Climate and Climate Change Data: An Overview. Digital Earth Summit on Geoinformatics 2008 Tools for Global Change Research ISDE08, 1999, 1999.

$\left[\mathrm{OLK}^{+} 14\right]$ Steffen Oeltze, Dirk J Lehmann, Alexander Kuhn, Gábor Janiga, Holger Theisel, and Bernhard Preim. Blood flow clustering and applications invirtual stenting of intracranial aneurysms. IEEE Transactions on Visualization and Computer Graphics, 20(5):686-701, 2014.

[PR99] R. Peikert and M. Roth. The "Parallel Vectors" operator-a vector field visualization primitive. Proceedings Visualization '99 (Cat. No.99CB37067), pages 263-532, 1999.

[RBS ${ }^{+}$18] Marc Rautenhaus, Michael Bottinger, Stephan Siemen, Robert Hoffman, Robert M. Kirby, Mahsa Mirzargar, Niklas Rober, and Rudiger Westermann. Visualization in Meteorology - A Survey of Techniques and Tools for Data Analysis Tasks. IEEE Transactions on Visualization and Computer Graphics, 24(12):3268-3296, 2018.

[RT12] Christian Rossl and Holger Theisel. Streamline Embedding for 3D Vector. IEEE Transactions on Visualization and Computer Graphics, 18(3):407-420, 2012.

[SANB ${ }^{+}$19] C. Schär, A. Arteaga, C Charpilloz N. Ban, S. di Girolamo, O. Fuhrer, T. Hoefler L. Hentgen, X. Lapillonne, D. Leutwyler, K. Osterried, D. Panosetti, S. Rüdisühli, 
L. Schlemmer, T. Schulthess, M. Sprenger, S. Ubbiali, and H. Wernli. Kilometerscale climate models: Prospects and challenges. Bulletin of the American Meteorological Society, 2019. submitted.

[SDS $\left.{ }^{+} 03\right] \quad$ J. Steppeler, G. Doms, U. Schättler, H. W. Bitzer, A. Gassmann, U. Damrath, and G. Gregoric. Meso-gamma scale forecasts using the nonhydrostatic model $\mathrm{lm}$. Meteorology and Atmospheric Physics, 82(1):75-96, Jan 2003.

[SFB ${ }^{+}$17] M. Sprenger, G. Fragkoulidis, H. Binder, M. Croci-Maspoli, P. Graf, C.M. Grams, P. Knippertz, E. Madonna, S. Schemm, B. Škerlak, and H. Wernli. Global Climatologies of Eulerian and Lagrangian Flow Features based on ERA-Interim. Bulletin of the American Meteorological Society, 98(8):1739-1748, 2017.

[SG16] Simon K. Siedersleben and Alexander Gohm. The Missing Link between TerrainInduced Potential Vorticity Banners and Banded Convection. Monthly Weather Review, 144(11):4063-4080, 2016.

[SGMM03] Edward J. Szoke, Ulrike Grote, Paula T. McCaslin, and Philip A. McDonald. D3D Update: Is it being used? In Proceedings of the 19th IIPS Conference, page 1.10, Long Beach, CA, 2003.

[SKA99] Roger C. Strawn, David N. Kenwright, and Jasim Ahmad. Computer Visualization of Vortex Wake Systems. AIAA Journal, 37(4):511-512, 1999.

[SKV ${ }^{+}$17] Christoph Schär, Grzegorz Kwasniewski, Hannes Vogt, Tarun Chadha, Thomas C. Schulthess, Xavier Lapillonne, Carlos Osuna, Daniel Lüthi, Oliver Fuhrer, David Leutwyler, and Torsten Hoefler. Near-global climate simulation at $1 \mathrm{~km}$ resolution: establishing a performance baseline on 4888 GPUs with COSMO 5.0. Geoscientific Model Development Discussions, pages 1-27, 2017.

[SSK10] Russ S. Schumacher, David M. Schultz, and John A. Knox. Convective snowbands downstream of the rocky mountains in an environment with conditional, dry symmetric, and inertial instabilities. Monthly Weather Review, 138(12):4416-4438, 2010 .

[SSL ${ }^{+}$03] Christoph Schär, Michael Sprenger, Daniel Lüthi, Qingfang Jiang, Ronald B. Smith, and Robert Benoit. Structure and dynamics of an Alpine potential-vorticity banner. Quarterly Journal of the Royal Meteorological Society, 129(588 PART B):825$855,2003$.

[STHW07] Kuangyu Shi, Holger Theisel, Helwig Hauser, and Tino Weinkauf. Path Line Attributes - an Information Visualization Approach to Analyzing the Dynamic Behavior of 3D Time-Dependent Flow Fields. TopoInvis, 2007.

[SW15] M. Sprenger and H. Wernli. The LAGRANTO Lagrangian analysis tool - Version 2.0. Geoscientific Model Development, 8(8):2569-2586, 2015.

[SW17] H. Saikia and T. Weinkauf. Global feature tracking and similarity estimation in time-dependent scalar fields. Computer Graphics Forum (Proc. EuroVis), 35(3):111, June 2017.

[SWTH07] Jan Sahner, Tino Weinkauf, Nathalie Teuber, and Hans Christian Hege. Vortex 
and strain skeletons in eulerian and Lagrangian frames. IEEE Transactions on Visualization and Computer Graphics, 13(5):980-989, 2007.

[TH07] J Gregory Trafton and Robert R Hoffman. Computer-Aided Visualization in Meteorology. Expertise out of Context: Proceedings of the 6th International Conference on Naturaistic Decision Making, pages 337-358, 2007.

[Tre02] L.A. Treinish. Task-specific visualization design: a case study in operational weather forecasting. 98:405-409,, 2002.

[TWHS03] Holger Theisel, Tino Weinkauf, Hans Christian Hege, and Hans Peter Seidel. Saddle Connectors - An Approach to Visualizing the Topological Skeleton of Complex 3D Vector Fields. Proceedings of the IEEE Visualization Conference, pages 225232, 2003.

[VCG18] Ambrogio Volonté, Peter A. Clark, and Suzanne L. Gray. The role of mesoscale instabilities in the sting-jet dynamics of windstorm Tini. Quarterly Journal of the Royal Meteorological Society, 144(712):877-899, 2018.

[Wik19] Wikipedia. Earth's rotation - Wikipedia, the free encyclopedia. http://en.wikipedia.org/w/index.php?title=Earth's\% 20 rotation\&oldid=893971188, 2019. [Online; accessed 25-April-2019]. 
Eidgenössische Technische Hochschule Zürich

Swiss Federal Institute of Technology Zurich

\section{Declaration of originality}

The signed declaration of originality is a component of every semester paper, Bachelor's thesis, Master's thesis and any other degree paper undertaken during the course of studies, including the respective electronic versions.

Lecturers may also require a declaration of originality for other written papers compiled for their courses.

I hereby confirm that I am the sole author of the written work here enclosed and that I have compiled it in my own words. Parts excepted are corrections of form and content by the supervisor.

Title of work (in block letters):

Extraction and Visual Analysis of Potential Vorticity Banners around the Alps

Authored by (in block letters):

For papers written by groups the names of all authors are required.

Name(s):

First name(s):

Bader

Robin

With my signature I confirm that

- I have committed none of the forms of plagiarism described in the 'Citation etiquette' information sheet.

- I have documented all methods, data and processes truthfully.

- I have not manipulated any data.

- I have mentioned all persons who were significant facilitators of the work.

I am aware that the work may be screened electronically for plagiarism.

Place, date

Zurich, 25.04.2019

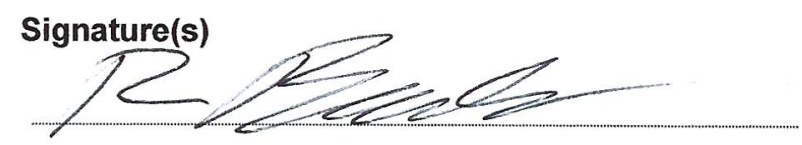

For papers written by groups the names of all authors are required. Their signatures collectively guarantee the entire content of the written paper. 